\title{
Micro-photoluminescence and micro-Raman spectroscopy of novel semiconductor nanostructures
}

\author{
Stanislav Filippov
}

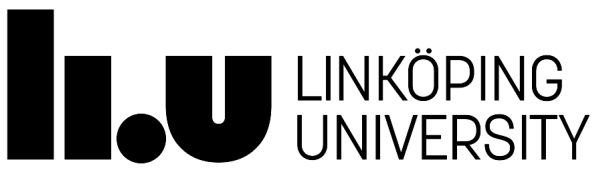

Functional electronic Materials Division

Department of Physics, Chemistry and Biology

Linköping University

SE-58183 Linköping, Sweden

Linköping 2016 
(C) Stanislav Filippov, unless otherwise is stated ISBN: 978-91-7685-877-6 ISSN: 0345-7524 


\section{Abstract.}

Low-dimensional semiconductor structures, such as one-dimensional nanowires (NWs) and zero-dimensional quantum dots (QDs), are materials with novel fundamental physical properties and a great potential for a wide range of nanoscale device applications. Here, especially promising are direct bandgap II-VI and III-V compounds and related alloys with a broad selection of compositions and band structures. For examples, NWs based on dilute nitride alloys, i.e. GaNAs and GaNP, provide both an optical active medium and well-shaped cavity and, therefore, can be used in a variety of advanced optoelectronic devices including intermediate band solar cells and efficient light-emitters. Self-assembled InAs QDs formed in the GaAs matrix are proposed as building blocks for entangled photon sources for quantum cryptography and quantum information processing as well as for spin light emitting devices. ZnO NWs can be utilized in a variety of applications including efficient UV lasers and gas sensors. In order to fully explore advantages of nanostructured materials, their electronic properties and lattice structure need to be comprehensively characterized and fully understood, which is not yet achieved in the case of aforementioned material systems. The research work presented this thesis addresses a selection of open issues via comprehensive optical characterization of individual nanostructures using micro-Raman ( $\mu$-Raman) and microphotoluminescence ( $\mu$-PL) spectroscopies.

In paper 1 we study polarization properties of individual GaNP and GaP/GaNP core/shell NWs using polarization resolved $\mu$-PL spectroscopy. Near band-edge emission in these structures is found to be strongly polarized (up to $60 \%$ at $150 \mathrm{~K}$ ) in the orthogonal direction to the NW axis, in spite of their zinc blende (ZB) structure. This polarization response, which is unusual for ZB NWs, is attributed to the local strain in the vicinity of the $\mathrm{N}$-related centers participating in the radiative recombination and to their preferential alignment along the growth direction, presumably caused by the presence of planar defects. Our findings therefore show that defect engineering via alloying with nitrogen provides an additional degree of freedom to control the polarization anisotropy of III-V nanowires, advantageous for their applications as a nanoscale source of polarized light.

Structural and optical properties of novel coaxial GaAs/Ga(N)As NWs grown on Si 
substrates, were evaluated in papers 2 -4. In paper 2 we show by using $\mu$-Raman spectroscopy that, though nitrogen incorporation shortens a phonon correlation length, the GaNAs shell with $[\mathrm{N}]<0.6 \%$ has a low degree of alloy disorder and weak residual strain. Additionally, Raman scattering by the GaAs-like and GaN-like phonons is found to be enhanced when the excitation energy approaches the $\mathrm{E}^{+}$transition energy. This effect was attributed the involvement of intermediate states that were created by N-related clusters in proximity to the $\mathrm{E}^{+}$subband. Recombination processes in these structures were studied in paper 3 by means of $\mu$-PL, $\mu$-PL excitation ( $\mu$-PLE), and time-resolved PL spectroscopies. At low temperatures, the alloy disorder is found to localize photoexcited carriers leading to predominance of localized exciton (LE) transitions in the PL spectra. Some of the local fluctuations in $\mathrm{N}$ composition are suggested to create threedimensional confining potentials equivalent to that for QDs, based on the observation of sharp PL lines within the LE contour. In paper 4 we show that the formation of these QD-like confinement potentials is somewhat facilitated in spatial regions of the NWs with a high density of structural defects, based on correlative spatially-resolved structural and optical studies. It is also concluded the principal axis of these QD-like local potentials is mainly oriented along the growth direction and emit light that is linearly polarized in the direction orthogonal to the NW axis. At room temperature, the PL emission is found to be dominated by recombination of free carriers/excitons and their lifetime is governed by non-radiative recombination via surface states. The surface recombination is found to become less severe upon $\mathrm{N}$ incorporation due to $\mathrm{N}$-induced modification of the surface states, possibly due to partial surface nitridation. All these findings suggest that the GaNAs/GaAs hetero-structures with the one-dimensional geometry are promising for fabrication of novel optoelectronic devices on foreign substrates (e.g. Si).

Fine-structure splitting (FSS) of excitons in semiconductor nanostructures has significant implications in photon entanglement, relevant to quantum information technology and spintronics. In paper 5 we study FSS in various laterally-arranged single quantum molecular structures (QMSs), including double QDs (DQDs), quantum rings (QRs), and QD-clusters (QCs), by means of polarization resolved $\mu$-PL spectroscopy. It is found that FSS strongly depends on the geometric arrangements of the QMSs, which can effectively tune the degree of asymmetry in the lateral confinement potential of the excitons and can reduce FSS even in a strained QD system to a limit similar to strain-free QDs. 
Fabrication of nanostructured $\mathrm{ZnO}$-based devices involves, as a compulsory step, deposition of thin metallic layers. In paper 6 we investigate impact of metallization by $\mathrm{Ni}$ on structural quality of $\mathrm{ZnO}$ NWs by means of Raman spectroscopy. We show that $\mathrm{Ni}$ coating of $\mathrm{ZnO}$ NWs causes passivation of surface states responsible for the enhanced intensity of the $\mathrm{A}_{1}(\mathrm{LO})$ in the bare $\mathrm{ZnO}$ NWs. From the resonant Raman studies, strong enhancement of the multiline Raman signal involving $\mathrm{A}_{1}(\mathrm{LO})$ in the $\mathrm{ZnO} / \mathrm{Ni} \mathrm{NW}$ s is revealed and is attributed to the combined effects of the Fröhlich interaction and plasmonic coupling. The latter effect is also suggested to allow detection of carbon-related species absorbed at the surface of a single $\mathrm{ZnO} / \mathrm{Ni} \mathrm{NW}$, promising for utilizing such structures as efficient nano-sized gas sensors. 


\section{Populärvetenskaplig sammanfattning.}

Lågdimensionella halvledarstrukturer såsom nanotrådar och kvantprickar är intressanta både ur ett rent vetenskapligt som ett praktiskt perspektiv, då de kan användas i en stor mängd applikationer, såsom nanoskaliga ljuskällor, gassensorer med mera. För nanoskaliga ljuskällor så är det ur ett tekniskt perspektiv speciellt önskvärt att de kan integreras med existerande Si-baserade elektroniska komponenter. Bland de mest lovande materialen för dessa applikationer finns de så kallade kväveutblandade legeringarna (dilute nitrides) såsom GaNP och GaNAs. Dessa legeringar har en väldig böjning av bandgapsenergin, vilket möjliggör bandgapsutformning genom att inkorporera en minimal mängd $\mathrm{N}$. Tillförsel av $\mathrm{N}$ tillåter vidare dessa material att kristallmässigt matcha $\mathrm{Si}$, vilket gör dessa material fördelaktiga för integrering med Si-elektronik. Endimensionella heterostrukturer med nanotrådar baserade på kväveutblandade legeringar blev tillgängliga mycket nyligen, vilket öppnade för möjligheten att producera axiella eller radiella heterostrukturer med önskvärda materialegenskaper. I denna avhandling visar vi att sådana GaNP/GaP och GaNAs/GaAs nanotrådar har utmärkt strukturell och optisk kvalitet och kan användas som effektiva ljuskällor i de teknologiskt utmanande gula respektive nära infraröda spektrala områdena. Vi visar också att legering med kväve tillåter förverkligandet av en hybridisering av noll- och endimensionella nanotrådstrukturer vilka fungerar som källor av starkt polariserat ljus. Resultaten av dessa studier finns sammanfattade i artiklar 1-4.

En annan typ av lågdimensionella halvledarstrukturer är kvantprickar. Föreslagna användningsområden av dessa nanostrukturer är bland annat som källor av kvantmekaniskt sammanflätade fotoner för kvantkryptografi, spinbaserade ljuskällor med mera. Självstrukturerande kvantprickar baserade på III-V-material är den mest vanliga typen av dessa nanostrukturer, på grund av den relativa enkelheten i den teknologiska processen. Finstrukturdelning av excitoner i dessa kvantprickar är en huvudsaklig parameter som har avgörande betydelse för sammanflätade fotoner och polarisation i konvertering mellan elektronspin och fotoner. I artikel 5 studerar vi olika lateralt arrangerade enskilda kvantmolekylära strukturer och visar att finstrukturdelning i dessa strukturer är starkt beroende av deras geometriska fördelning, vilket effektivt kan reducera finstrukturdelningen i ett system med uttöjda kvantprickar så långt som till en gräns lik den hos outtöjda kvantprickar. Denna ansats ger en ny väg att skapa högsymmetriska kvantmekaniska ljuskällor som är önskvärda för förverkligandet av kvantmekaniskt 
sammanflätade fotoner och för spintronikenheter baserade på sådana nanostrukturer.

Slutligen, nanostrukturer baserade på ZnO är för nuvarande sedda som ett av de huvudsakliga materialsystemen för optoelektroniska tillämpningar och gassensorer. Både egenskaper som gassensor och effektivitet som ljuskälla i ZnO kan vidare ökas genom addering av en tunn metallfilm. För att få en djup förståelse av dessa effekter så undersöks i artikel 6 effekter av beläggning med Ni på strukturella egenskaper hos ZnO nanotrådar. 


\section{Preface.}

This thesis summarize my research done during the period of 2011-2016 of 4.5 years of PhD studies in the Division of Functional Electronic Materials at Department of Physics, Chemistry and Biology (IFM), Linköping University, Sweden.

The thesis are divided into two parts. The first part contains literature review of related topics in semiconductor physics and modern advances in relevant research areas together with description of experimental techniques. The second part contains papers which represent the research results. 


\section{List of publications. \\ Paper 1.}

Origin of Strong Photoluminescence Polarization in GaNP Nanowires.

S. Filippov, S. Sukrittanon, Y. Kuang, C. W. Tu, P. O. Å. Persson, W. M. Chen, and I. A. Buyanova. Nano lett. 14, 5264 (2014).

\section{Paper 2.}

Structural properties of GaNAs nanowires probed by micro-Raman spectroscopy.

S. Filippov, F. Ishikawa, W. M. Chen, and I. A. Buyanova. Semicond. Sci. Technol. 31, 025002 (2016).

\section{Paper 3.}

Origin of radiative recombination and manifestations of localization effects in GaAs/GaNAs core/shell nanowires.

S. L. Chen, S. Filippov, F. Ishikawa, W. M. Chen, and I. A. Buyanova. Appl. Phys. Lett. 105, 253106 (2014).

\section{Paper 4.}

Polarization resolved studies of ultra-narrow transitions in GaAs/GaNAs core/shell nanowires.

S. Filippov, M. Jansson, J. E. Stehr, J. Palisaitis, P. O. Å. Persson, F. Ishikawa, W. M Chen, I. A. Buyanova. Submitted manuscript.

\section{Paper 5.}

Exciton Fine-Structure Splitting in Self-Assembled Lateral InAs/GaAs QuantumDot Molecular Structures.

S. Filippov, Y. Puttisong, Y. Huang, I. A. Buyanova, S. Suraprapapich, C. W. Tu, and W. M. Chen. ACS Nano 9, 5741 (2015). 


\section{Paper 6.}

Effects of Ni-coating on $\mathrm{ZnO}$ nanowires: A Raman scattering study.

S. Filippov, X. J. Wang, M. Devika, N. Koteeswara Reddy, C. W. Tu, W. M Chen, and I. A. Buyanova. J. Appl. Phys. 113, 214302 (2013).

\section{My contributions to the papers.}

Papers 1, 2, and 6. I have performed all optical measurements and data analysis, interpreted data together with my co-authors and wrote the first version of manuscript.

Paper 3. I have performed $\mu$-PL, $\mu$-PLE and temperature dependent $\mu$-PL measurements on individual NW, participated in data analysis, and interpreted data together with my co-authors.

Papers 4 and 5. I have performed most of the optical measurements, analyzed and interpreted data together with my co-authors and wrote the first version of the manuscript.

\section{Papers not included in the thesis.}

Evidence for coupling between exciton emissions and surface plasmon in $\mathrm{Ni}$ coated $\mathrm{ZnO}$ nanowires.

Q. J. Ren, S. Filippov, S. L. Chen, M. Devika, N. Koteeswara Reddy, C. W. Tu, W.

M. Chen, and I. A. Buyanova. Nanotechnology 23, 425201 (2012).

Defects in $\mathrm{N}, \mathrm{O}$ and $\mathrm{N}, \mathrm{Zn}$ implanted $\mathrm{ZnO}$ bulk crystals.

J. E. Stehr, X. J. Wang, S. Filippov, S. J. Pearton, I. G. Ivanov, W. M. Chen, and I. A. Buyanova. J. Appl. Phys. 113, 103509 (2013).

Defect properties of $\mathrm{ZnO}$ nanowires revealed from an optically detected magnetic resonance study.

J. E. Stehr, S. L. Chen, S. Filippov, M. Devika, N. Koteeswara Reddy, C. W. Tu, W. M. Chen, and I. A. Buyanova. Nanotechnology 24, 015701 (2013). 


\section{Acknowledgments.}

I would like express my gratitude to professors Irina Buyanova and Weimin Chen for giving me the opportunity to work in their group. I want thank you for all inspiring discussions and efforts to teach me many things. I learned a lot during these years.

I would also express my gratitude to my current and former group colleagues for great time during and, what is very valuable, after work: Dr. J. Stehr, Dr. A. Dobrovolsky, Dr. Y. Puttisong, Dr. S. Chen, Dr. D. Dagnelund, Y. Hung, M. Jansson. Special thanks to Dr. J. Stehr for your help in the lab, fruitful discussions regarding and not regarding the work.

I want thank my parents for their endless help and care. The reader could have been reading totally different thesis without your support.

I also acknowledge my numerous friends, who are too many to list here, for the great time, company and sometimes some help. Special thanks to M. Chubarov, for great time during all these years; S. Samarkin for great company and help with the car; S. Schimmel for great time and company. 


\section{Contents}

Chapter 1 Basic crystal and electronic structure. ..................................................... 1

1.1. Crystal structure of semiconductors.................................................................. 1

1.2. Electronic structure of bulk semiconductors. ......................................................... 4

1.2.1. Band formation......................................................................................... 4

1.2.2. Band structure of zinc blende and wurtzite semiconductors........................ 7

1.3. Strain in zinc blende semiconductors. .............................................................

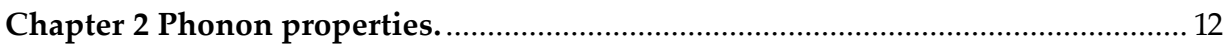

2.1. Lattice dynamics in harmonic approximation. ................................................. 12

2.1.1. Equation of motion of oscillating 3D lattice in harmonic approximation. . 12

2.1.2. Normal coordinates and symmetry of lattice oscillations. .......................... 14

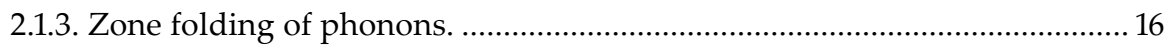

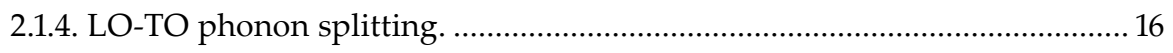

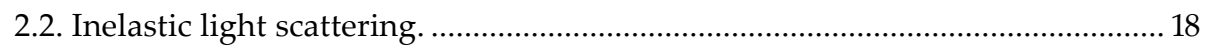

2.2.1. Simple classical treatment. ........................................................................ 18

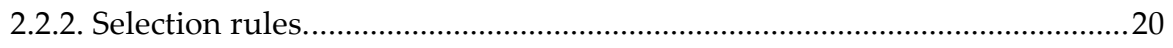

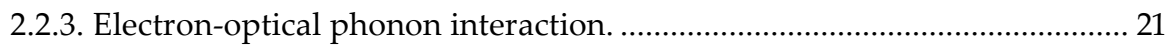

2.3. Strain and alloying effects on Raman spectrum.............................................. 21

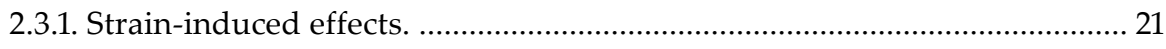

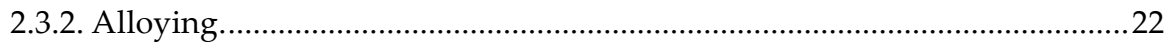

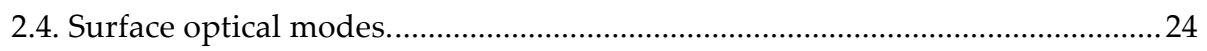

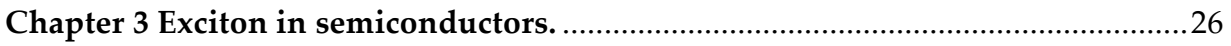

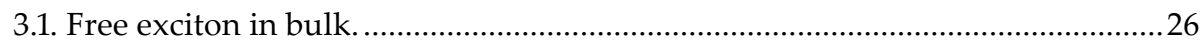

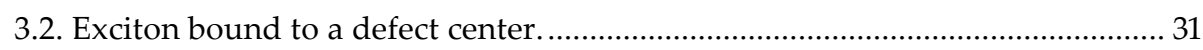

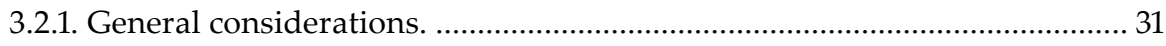

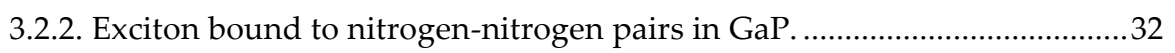

3.3. Exchange interaction in zero dimensional semiconductor structures............... 35

Chapter 4 Polarization properties of semiconductor nanowires. ............................. 40

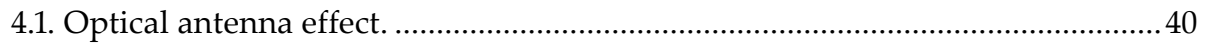

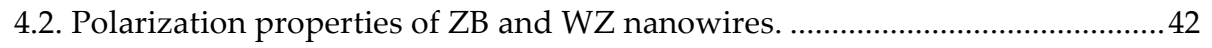

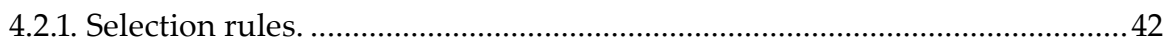

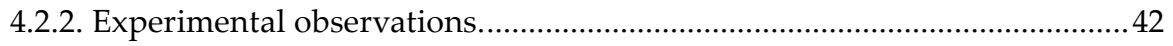

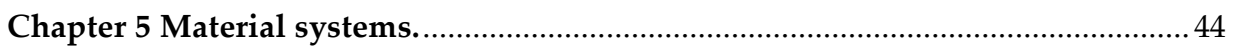

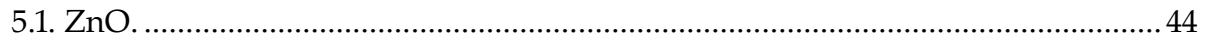

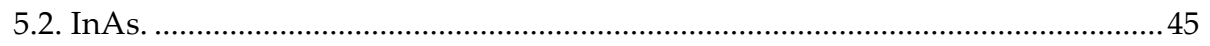

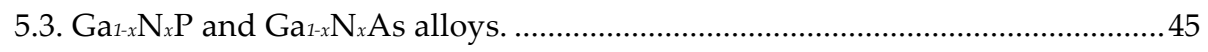




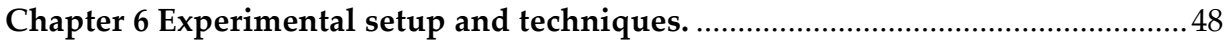

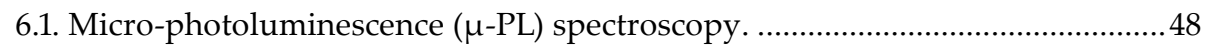

6.2. Micro-photoluminescence excitation spectroscopy ...........................................49

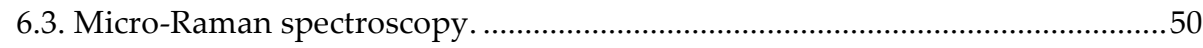

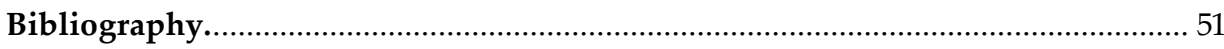




\section{Chapter 1 Basic crystal and electronic structure.}

\subsection{Crystal structure of semiconductors.}

A description of a crystal structure is so called Bravais lattices. A Bravais lattice can be defined as set of points which positions are given by vector $\mathbf{R}$ of the form:

$$
\mathbf{R}=n_{1} \mathbf{a}_{1}+n_{2} \mathbf{a}_{2}+n_{3} \mathbf{a}_{3}
$$

here $n_{1}, n_{2}, n_{3}$ are integer positive numbers, and $\mathbf{a}_{1}, \mathbf{a}_{2}, \mathbf{a}_{3}$ are three any non-coplanar vectors. It is obvious that set of vectors $\mathbf{a}_{i}$ is not unique. These vectors are called primitive vectors and are said to generate (span) the lattice.

A volume of space containing only one Bravais lattice point such that it fills all of space when translated through the vectors $\mathbf{R}$ without either overlapping itself or leaving voids is called primitive cell. The choice of the primitive cell is also not unique though its volume does not depend on the chosen cell geometry. It should be noted that the primitive cell does not necessary satisfy all the symmetry operations of a crystal.

(a)

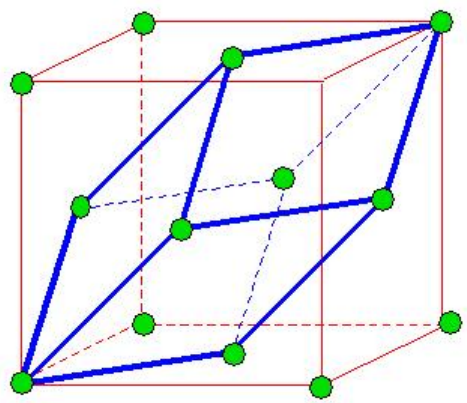

(b)

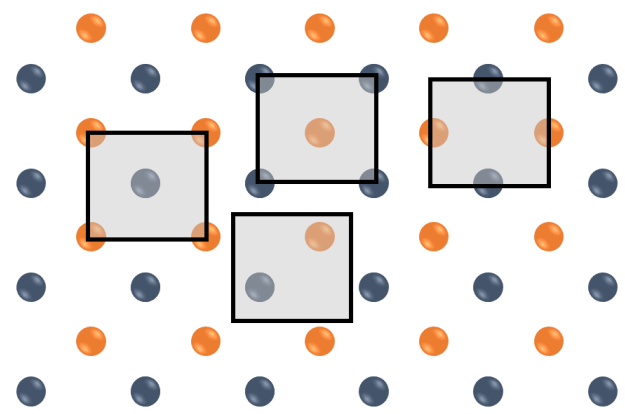

Figure 1. a) A unit cell of the fcc lattice and the primitive lattice [1]. b) different options to choose a basis

Alternatively one can fill-up the space with a nonprimitive cell known as unit cells or conventional unit cells. The unit cell fills the space without voids or overlapping with itself upon translation through a subset of $\mathbf{R}$. The unit cell is generally larger than the primitive cell and contains more than one lattice point but has advantage of possessing the symmetry of a crystal. Figure 1a shows an example of the unit and the primitive cells of the face-centered cubic (fcc) lattice. The fcc lattice consists of points located at the eight corners of the cube and centered on its six facets. 
A real crystal can be described by "attaching" to the Bravais lattice a basis. The basis describes arrangement of atoms within one primitive cell. Position of each atom in the basis is given through the primitive vectors $\mathbf{a}_{1}, \mathbf{a}_{2}, \mathbf{a}_{3}$. In the aforementioned fcc lattice one can choose a four-point basis

$$
0, \frac{a}{2}(\mathbf{x}+\mathbf{y}), \frac{a}{2}(\mathbf{y}+\mathbf{z}), \frac{a}{2}(\mathbf{z}+\mathbf{x})
$$

where $a$ is the lattice constant and $\mathbf{x}, \mathbf{y}, \mathbf{z}$ are primitive vectors along $x, y, z$ axes. With this basis the fcc lattice can be described as a simple cubic lattice with points at the edges of the cube. In reality the choice of basis is also not unique and can be performed in several ways as demonstrated in figure $1 b$.

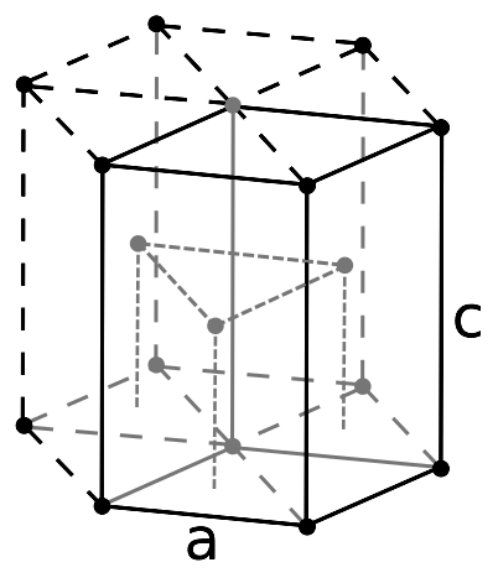

Figure 2. Hexagonal close-packed structure. The inner grey triangle depicts part of another triangular net [2].
Many practically important semiconductors crystallize in diamond-like lattice. This lattice is constructed from two interpenetrating fcc lattices shifted along the body diagonal by $1 / 4$ of its length. It can be regarded as an fcc lattice with a twopoint basis $0,(a / 4)(\mathbf{x}+\mathbf{y}+\mathbf{z})$. Example of semiconductors crystallizing into the diamond-like lattice is $\mathrm{Si}$ and Ge. GaAs and other cubic III-V materials crystallize in the same structure, but since they consist of two different atoms, the resulting structure lacks center of inversion and is named zinc-blende $(\mathrm{ZB})$ structure.

Another important class of crystal structures is called hexagonal close-packed (hcp) structure schematically shown on Figure 2. It is not Bravais lattice and is obtained from simple hexagonal lattice by stacking the triangular nets on top of each other. The structure is characterized by set of two lattice parameters $a$ and $c$ shown on figure 2 . In the ideal hcp structure the ratio between $a$ and $c$ is $c / a=\sqrt{8 / 3}$. Semiconductors like ZnO or hexagonal GaN crystallize in the so-called wurtzite (WZ) structure that consists of two interpenetrating hcp lattices in which each sublattice is occupied by atoms of the same kind. It should be noted that $\mathrm{WZ}$ and $\mathrm{ZB}$ structures differ in the sequence of atomic planes - see Figure 3. 

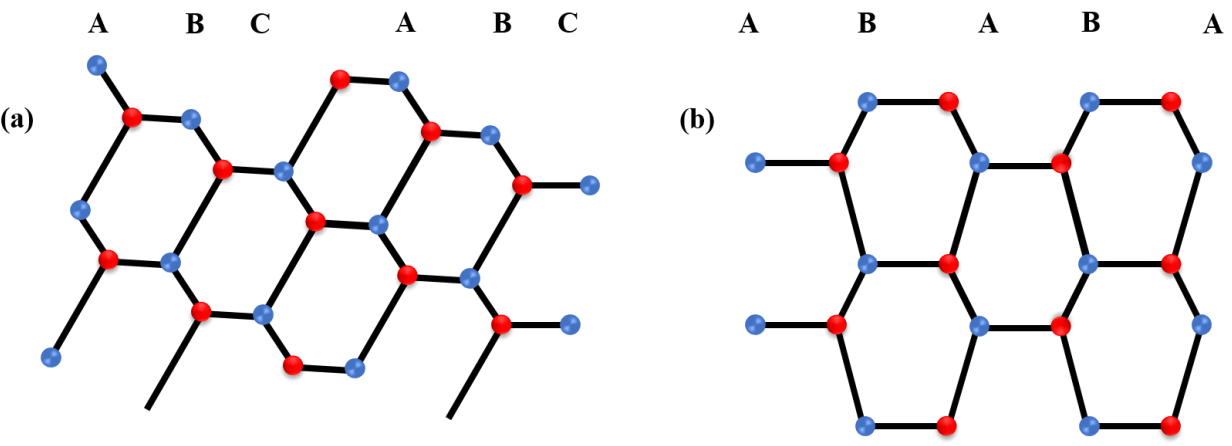

Figure 3. Schematic illustration of the $Z B$ and $W Z$ sequence of atomic planes stacking. (a) ZB lattice; (b) WZ lattice. The crystallographic directions are introduced below.

An orientation of a crystal plane can be described by a set of 3 integer numbers known as Miller indexes. The Miller indexes are defined as following: i) we first find primitive vectors $\mathbf{a}_{1}, \mathbf{a}_{2}, \mathbf{a}_{3}$, which define intercepts of the crystal plane and the crystal axes; ii) we then take the reciprocals of these numbers and reduce them to the smallest integers that preserve the same relation as the reciprocals. The result is denoted as $(h k l)$. The crystal direction orthogonal to the $(h k l)$ plane is denoted as [hkl]. The set of crystallographically equivalent directions is denoted as $\langle h k l\rangle$. If crystal planes in hexagonal systems are indexed using Miller indices, then crystallographically equivalent planes have indices which appear dissimilar. This can be overcome using the so called Miller-Bravais system, where an additional $4^{\text {th }}$ axis directed at 120 degree relative to the $\mathbf{x}$ and $\mathbf{y}$ axes is introduced in the $(x y)$ plane. The plain is then specified with four indexes ( $h k i l)$ where the third index is negative and is equal to the sum of the first two.

A very important concept is the concept of reciprocal lattice constructed from vectors of reciprocal length as:

$$
\mathbf{b}_{1}=2 \pi \frac{\mathbf{a}_{2} \times \mathbf{a}_{3}}{\mathbf{a}_{1} \cdot\left(\mathbf{a}_{2} \times \mathbf{a}_{3}\right)}
$$

The rest of the vectors is obtained by cyclic permutations. In another words the reciprocal lattice is constructed from vectors of reciprocal length. The reciprocal lattice is crucial for description of numerous physical properties of a solid such as band structure, lattice vibrations, etc. 


\subsection{Electronic structure of bulk semiconductors.}

\subsubsection{Band formation.}

A free electron wavefunction is a plane wave of the form $\Psi=e^{i \mathbf{k r}}$ with the wave vector $\mathbf{k}$. Because of periodical arrangement of constituent atoms in solids, an electron in solids moves in an effective crystal potential $U(\mathbf{r})$, which should be invariant under translation by a lattice vector $\mathbf{a}_{n}: U(\mathbf{r})=U\left(\mathbf{r}+\mathbf{a}_{n}\right)$. Without knowing the exact electronic structure it can be shown that the electronic wavefuntion in the crystal field can be written as $\psi(\mathbf{r})=e^{i \mathbf{k r}} u_{\mathbf{k}}(\mathbf{r})$, where the function $u_{\mathbf{k}}(\mathbf{r})$ is periodic with periodicity of the lattice. In another words, the electron wave function in the crystal is a product of the plane wave and the function with the lattice periodicity. This statement is Bloch theorem.

Electronic states of atoms in solids form energy bands. Their formation can be understood within the tight-binding (TB) method. The position of an atom in a primitive cell can be expressed as

$$
\mathbf{r}_{j}=\mathbf{R}_{m}+\mathbf{r}_{\alpha}
$$

where $\mathbf{R}_{m}$ denotes position of the $\mathrm{m}^{\text {th }}$ primitive cell and $\mathbf{r}_{\alpha}$ denotes position of $\alpha^{\text {th }}$ atom the cell. Let assume that the solution of Schrodinger equation for a single isolated atom

$$
H \Psi_{k}(\mathbf{r})=\left[-\frac{\hbar^{2}}{2 m} \nabla^{2}+V(\mathbf{r})\right] \Psi_{k}(\mathbf{r})=E(\mathbf{k}) \Psi_{k}(\mathbf{r})
$$

is known with atomic orbitals $\phi_{j}\left(\mathbf{r}_{\alpha}\right)$. They are known as Löwdin orbitals and constructed in the way that they are orthogonal to each other when they belong to atoms located at different positions. Then the Bloch functions can be formed from the atomic orbitals as:

$$
\psi_{\mathbf{k}}^{\alpha, j}=1 / \sqrt{N} \sum_{m} \exp \left(i \mathbf{k} \mathbf{R}_{\mathbf{m}}\right) \phi_{j}\left(\mathbf{r}-\mathbf{R}_{\mathbf{m}}-\mathbf{r}_{\alpha}\right)
$$

where $\mathrm{N}$ is the number of primitive cells. Consequently the TB method assumes that the crystal's electronic wave functions can be written as a liner combination of the Bloch functions:

$$
\Psi_{k}=\sum_{m, \alpha} C_{m, \alpha} \psi_{\mathbf{k}}^{\alpha, j}
$$

Substituting the expression (1.7) of the $\Psi_{\mathbf{k}}$ into the Schrödinger equation of an electron in periodic crystal field and multiplying by $\Psi_{\mathbf{k}}^{*}$, one obtains the set of linear equations for $C_{m, \alpha}$ : 


$$
\sum_{m . l}\left(H_{m l, m^{\prime} l^{\prime}}-E_{k} \delta_{m m^{\prime}} \delta_{l l^{\prime}}\right) C_{m^{\prime} l^{\prime}}(\mathbf{k})=0
$$

The energy dispersion and the electronic wave functions are obtained through the diagonalization of $H_{m l, m \prime l}$. The key concept of the TB method is an overlap of the orbitals $\psi_{\mathbf{k}}^{\alpha, j}$ in (1.6). The overlap (bonding) between the orbitals can occur by the head-on overlap and this type of bonding is called $\sigma$-bonding. The overlap can also happen by sides, and this type of boding is called $\pi$-bonding. Many important semiconductors such as $\mathrm{Si}$ or GaAs are formed from materials with $s$ and $p$ valence electrons. As a result, the orbitals mix and form four equivalent orbitals, so-called $s p^{3}$ hybrids. Each atom in such structure is surrounded by 4 neighbors (say one Ga atom is surrounded by 4 As atoms and vice versa) located at the corners of a tetrahedron. Therefore these semiconductors are called tetrahedral. Under assumption of nearest-neighbor interactions, the coupling between orbitals can be defined by four basic overlap parameters (in case when the lattice is formed by one type of atoms), or 8 parameters for binary compounds such as GaAs. These include: the $\sigma$-bonding between the s-orbitals $\left(V_{s S \sigma}\right)$, the $\sigma$-bonding between the $s$ - and $p$-orbital $\left(V_{s p \sigma}\right)$, the $\sigma$-bonding between two p-orbitals $\left(V_{p p \sigma}\right)$, and the $\pi$-bonding between two p-orbitals $\left(V_{p p \pi}\right)$. These are the only TB "fitting" parameters, which are used to calculate band structure. In case of $s p^{3}$ hybridization and the assumption of nearest-neighbor interactions, diagonalization of $H_{m l, m \prime l}$, is described in details in [3]-[5].

The Hamiltonian is diagonalized in the basis of bonding and antibonding orbitals. The band formation in tetrahedral semiconductors can be qualitatively understood in the following way. The atomic orbitals mix and couple with each other. The coupling results in the formation of bonding and antibonding orbitals with the bonding states being lower in energy. Upon bonding, each atom in the crystal is connected to all other crystal atoms via bonding with its neighbors. As a result, the discrete atomic orbitals are broadened into the energy bands. At $0 \mathrm{~K}$ the highest bonding states are fully occupied and comprise the valence band (VB), whereas the lowest antibonding states are completely empty and comprise the conduction band (CB). The energy splitting between these states is the bandgap. The strength of the bonding-antibonding splitting is determined by the overlapping integrals. They increase when the interatomic distance $d$ is decreased. The magnitude of the overlapping integrals scales as $d^{-2}$. Therefore, the highly ionic materials like $\mathrm{ZnO}$ with a typical lattice constant of $3 \AA$ have a much higher bandgap than III-V cubic semiconductors with typical lattice constants of 5-6 $\mathrm{A}$ [5]. 
Electron energy $E$ in the band is a function of its momentum $\mathbf{p}$ and the corresponding relation defines the energy dispersion $E=E(\mathbf{p})$. Close to extrema points the $E(\mathbf{p})$ can be expanded as:

$$
E(\mathbf{p})=E\left(\mathbf{p}_{o}\right)+\frac{1}{2} \frac{\partial^{2} E(\mathbf{p})}{\partial p_{\alpha} \partial p_{\beta}} \times\left(p_{0}-p_{\alpha}\right)\left(p_{0}-p_{\beta}\right)+\cdots
$$

where $\mathbf{p}_{\alpha \beta}$ are the momentum components and $\mathbf{p}_{0}$ is the electron momentum at the band minimum (maximum). The momentum of an electron in a crystal is defined as $\mathbf{p}=\hbar \mathbf{k}$, where $\mathbf{k}$ is the wave vector. The parameter $m_{\alpha \beta}$ is introduced as $\left(m^{-1}\right)_{\alpha \beta}=\frac{1}{\hbar^{2}} \times \frac{\partial^{2} E(\mathbf{k})}{\partial k_{\alpha} \partial k_{\beta}}$, where $\mathbf{k}_{\alpha \beta}$ are wave vector components. The tensor $m_{\alpha \beta}$ has dimensionality of a mass and is called effective mass tensor. As any tensor, $m_{\alpha \beta}$ can be transformed to a diagonal form. Keeping only the $2^{\text {nd }}$ order terms, the expression (1.9) can then simplified as:

$$
E(\boldsymbol{k})=E\left(\mathbf{k}_{0}\right)+\frac{1}{2 \hbar^{2}} \sum_{\alpha=x, y, z} m_{\alpha \beta}^{-1}\left(k_{0, \alpha}-k_{\alpha}\right)^{2}
$$

In case of the isotropic effective mass $m^{*}$, the equation (1.10) acquires the well-known form $E(\mathbf{k})=E\left(\mathbf{k}_{0}\right)+\frac{\hbar^{2} k^{2}}{2 m^{*}}$.

In the picture above we didn't consider electron spin which gives rise to electron's magnetic moment. For a free electron spin and the magnetic moment are related by $\boldsymbol{\mu}=-\frac{|e|}{m c} \frac{\hbar}{2} \frac{\mathbf{S}}{|\mathbf{S}|}$. Thus an electron in an atom sees magnetic field of its own orbital motion and couples to it. This coupling is named spin-orbit coupling and is a relativistic effect. The conduction band states are formed from s-orbitals, and their angular moment $l=0$. Therefore these states do not experience the spin-orbit interaction. However the valence band edge states are formed of $p$-states for which $l=1$ and thus these states experience spin-orbit interaction. Generally speaking the spin-orbit interaction partly lifts degeneracy of some of valence band edge states. The situation is complicated by crystal symmetry.

The symmetry properties of a crystal lattice are described in terms of a point space group. Every element of a space point group can be written as a product of an element from the point group and some translation. When the spin-orbit interaction is included, the wave function contains spatial and spin parts. Therefore, the irreducible representation of a state depends on the spin angular momentum. The transformation properties are now described by the so-called double space group. The elements of a double group 
are obtained by a direct product of the symmetry elements of the group and the irreducible representation of the spin function often denoted in literature as $D_{1 / 2}$ (basically, spinor). More on properties of double groups can be found in [6]. We will discuss effects of symmetry and spin-orbit interaction on the band structure near the $\Gamma$ point in the next section.

\subsubsection{Band structure of zinc blende and wurtzite semiconductors.}

A majority of optical processes involve zone center electrons, so the band structure near the $\Gamma$ point is of a particular interest. Let us start with the zinc blende structure, which belongs to the $\mathrm{T}_{\mathrm{d}}$ space group. The conduction band is formed from fully symmetrical s-like wave functions. Therefore, they should transform as completely symmetrical representation $\Gamma_{1}$. The valence band edge states are formed from $p$-orbitals, and under the symmetry operations of the group they transform as a 3-dimensional vector. The $\mathrm{T}_{\mathrm{d}}$ group contains only one irreducible representation which transforms as a vector, namely, $\Gamma_{4}$. Thus without taking into account spin-orbit interaction, band symmetries at the $\Gamma$ point are $\Gamma_{1 c}$ and $\Gamma_{4 v}$, where the subscripts $c$ and $v$ stands for conduction and valence bands respectively [7]-[9].

When electron spin is under consideration, the irreducible representations of the conduction band and valence band states are transformed as [6], [10]:

$$
\begin{aligned}
& \Gamma_{1} \otimes D_{1 / 2}=\Gamma_{6} \\
& \Gamma_{4} \otimes D_{1 / 2}=\Gamma_{7} \oplus \Gamma_{8}
\end{aligned}
$$

Thus the degenerate in absence of spin-orbit interaction valence band becomes split into 2 bands. The basis functions for these new irreducible representations are obtained by taking the direct product of the basis functions of the corresponding irreducible representations. For example, in order to obtain the basis functions of $\Gamma_{7} \oplus \Gamma_{8}$, we should find the result of direct product of basis functions of $\Gamma_{4}$ and $\mathrm{D}_{1 / 2}$ (or $\Gamma_{6}$ in $\mathrm{T}_{d}^{2}$ group). The results are given in [10] as coupling coefficients. By doing so, one obtains the hole basis functions of a ZB semiconductor:

$\Gamma_{8} \operatorname{set}(J=3 / 2)$

$$
\begin{gathered}
\left|j=\frac{3}{2}, m=\frac{3}{2}\right\rangle=-\frac{1}{\sqrt{2}}|X+i Y\rangle|\uparrow\rangle \\
\left|J=\frac{3}{2}, m=\frac{1}{2}\right\rangle=-\frac{1}{\sqrt{6}}(|X+i Y\rangle|\downarrow\rangle-2|Z\rangle|\uparrow\rangle) \\
\left|J=\frac{3}{2}, m=-\frac{1}{2}\right\rangle=\frac{1}{\sqrt{6}}(|X-i Y\rangle|\uparrow\rangle+2|Z\rangle|\downarrow\rangle)
\end{gathered}
$$




$$
\left|J=\frac{3}{2}, m=-\frac{3}{2}\right\rangle=\frac{1}{\sqrt{2}}|X-i Y\rangle|\downarrow\rangle
$$

$\Gamma_{7} \operatorname{set}(J=1 / 2)$

$$
\begin{gathered}
\left|J=\frac{1}{2}, m=\frac{1}{2}\right\rangle=\frac{1}{\sqrt{3}}(|X+i Y\rangle|\downarrow\rangle+|Z\rangle|\uparrow\rangle) \\
\left|J=\frac{1}{2}, m=-\frac{1}{2}\right\rangle=\frac{1}{\sqrt{3}}(|X-i Y\rangle|\uparrow\rangle-|Z\rangle|\downarrow\rangle)
\end{gathered}
$$

Here $J$ is the eigenvalue of the total angular momentum operator $\mathbf{J}=\mathbf{L}+\mathbf{S}, m$ is its projection, and $|X\rangle,|Y\rangle, \mid Z>$ are the three degenerate valence band states (in absence of the spin-orbit interaction)formed from the bonding states of $p_{x}, p_{y}$, and $p_{z}$ orbitals. And $|\uparrow\rangle$, $\mid \downarrow>$ are spin-up and spin-down states.

Thus we see that the spin-orbit interaction partially lifts the degeneracy of the valence band and results in splitting between $J=3 / 2$ and $J=1 / 2$ states. It can be shown that $J=1 / 2$ states lay higher in energy that $J=3 / 2$ states [5], [6]. The hole states with $J_{z}= \pm 3 / 2$ are so called heavy hole states since their effective mass is higher than those of $J_{z}= \pm 1 / 2$ named light hole states.

Treatment of wurtzite crystal structure is done in similar way. The CB symmetry becomes $\Gamma_{1} \otimes D_{1 / 2}=\Gamma_{7}$. The $\Gamma_{4 v}$ irreducible representation in $\mathrm{T}_{\mathrm{d}}$ group is three-dimensional, however, there are no three-dimensional irreducible representations in the $\mathrm{C}_{6 \mathrm{v}}$ group. Thus, this representation is obviously reducible, and the valence band must split. The resulting irreducible representations of the valence subbands are obtained following a standard decomposition procedure. As a result, the $\Gamma_{4 v}$ band in WZ crystals split into two bands of $\Gamma_{5 v}$ and $\Gamma_{1 v}$ symmetries [7]-[9]. Since $\Gamma_{5 v}$ belongs to the two-dimensional representation, the lowering of crystal symmetry from $\mathrm{T}_{\mathrm{d}}$ to $\mathrm{C}_{6 \mathrm{v}}$ decouples $p_{x}, p_{y}$ from $p_{z}$ states (which form the $\Gamma_{1 v}$ states). This effect is often named in the literature as crystal field splitting. In the presence of spin-orbit interaction the $\Gamma_{5 v}$ states further split into the $\Gamma_{7 v}$ and $\Gamma_{9 v}$ states. Thus the valence band consists of three bands, conventionally referred to as $\mathrm{A}, \mathrm{B}$, and $\mathrm{C}$ subbands. We summarize the results in Figure 4. 


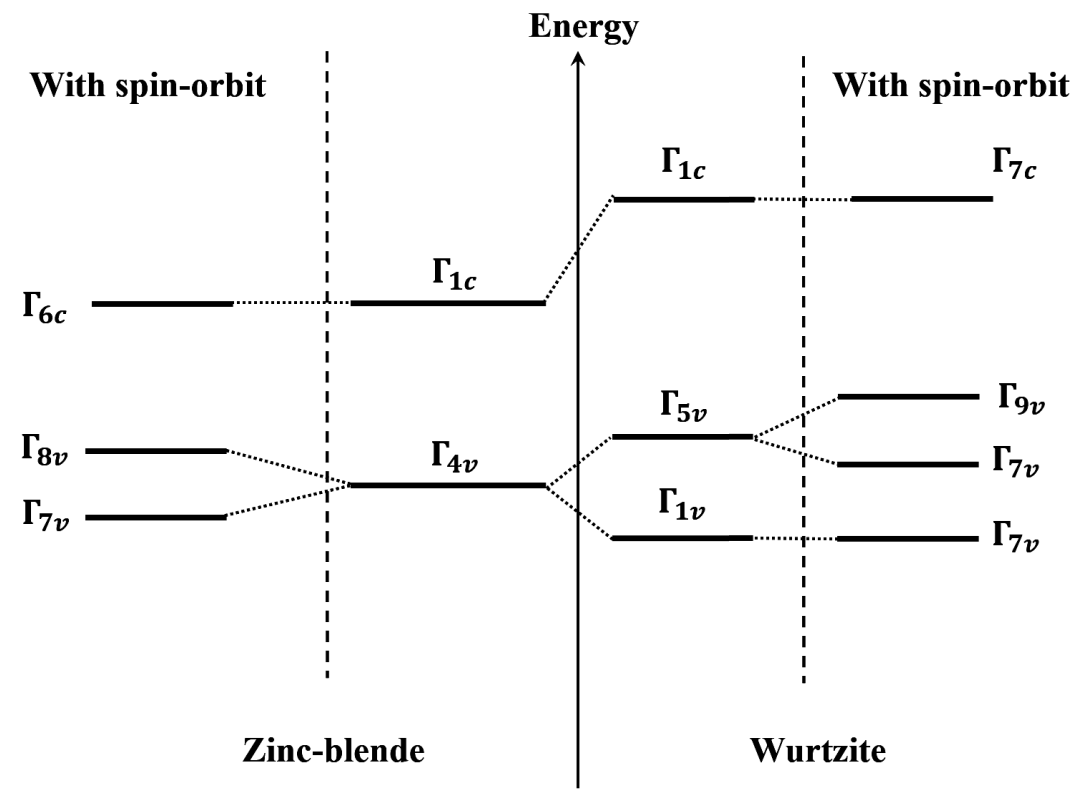

Figure 4. Schematic of the band energies at $\Gamma$ point.

\subsection{Strain in zinc blende semiconductors.}

When a crystal experiences external forces, the atoms in the lattice change their equilibrium positions. In case of small deformations the strain can be defined as

$$
\epsilon_{\alpha, \beta}=\frac{1}{2}\left(\frac{\partial u_{\alpha}}{\partial x_{\beta}}+\frac{\partial u_{\beta}}{\partial x_{\alpha}}\right)
$$

where $u_{\alpha}$ is the displacement of the lattice point, $x_{\beta, \alpha}$ are directions, and $\alpha, \beta=x, y, z$. In general case $\epsilon_{\alpha, \beta}$ is a tensor. Due to symmetrical definition of the strain tensor, it has less than 9 independent components. Thus often the strain tensor is replaced by a vector $\mathbf{e}$ defined as

$$
\begin{aligned}
& e_{x x}=\epsilon_{x x} ; e_{y y}=\epsilon_{y y} ; e_{z z}=\epsilon_{z z} \\
& e_{x y}=\epsilon_{x y}+\epsilon_{y x} ; \\
& e_{y z}=\epsilon_{y z}+\epsilon_{z y} ; \\
& e_{z x}=\epsilon_{z x}+\epsilon_{x z}
\end{aligned}
$$

and

$$
\mathbf{e}=\left(e_{x x} ; e_{y y} ; e_{z z} ; e_{y z} ; e_{z x} ; e_{x y}\right)
$$

The components like $e_{x x}$ describe distortions associated with change of the material's 
volume and components like $e_{x y}$ define changes of angles between the lattice basis vectors.

The deformation of the crystal lattice results in the appearance in forces inside the material. Stress is defined as the force per unit area that appears in response on the external forces. In case of not very high stress, the stress is linearly related to the strain, and this relation is known as Hooke's law as:

$$
\sigma_{i j}=\sum_{\alpha \beta} C_{i j \alpha \beta} \epsilon_{\alpha \beta} ; i, j, \alpha, \beta=x, y, z
$$

or $\sigma_{i}=\sum_{n} C_{i n} e_{n}$, where $C_{i n}=\left(C_{x x}, C_{y y}, C_{z z}, C_{y z}, C_{z x}, C_{x y}\right)$

The coefficients $C_{i j \alpha \beta}$ are called elastic stiffness constants and form a $4^{\text {th }}$ rank tensor. Crystal symmetry implies limitations on number of independent components. For example, in cubic crystals $C_{i n}$ has only 3 independent components.

The relation between stress and strain is given by the compliance tensor $S_{i j \alpha \beta}$ :

$$
\epsilon_{i j}=\sum_{\alpha \beta} S_{i j \alpha \beta} \sigma ; i, j, \alpha, \beta=x, y, z
$$

In cubic crystals the compliance and the stiffness tensors have the same form and number of independent components $C_{i n}$ and $S_{\text {in }}$ respectively.

Application of stress results in changes of the atomic positions. In crystals lacking a center of inversion, such as cubic III-V compounds, the application of stress results in appearance of non-vanishing macroscopic polarization and, therefore, an electric field. This effect is known as the piezoelectric effect. The polarization P relates to the strain through the piezoelectric tensor as:

$$
P_{i}=\sum_{j k} \pi_{i j k} \epsilon_{j k}
$$

or

$$
P_{i}=\sum_{j} e_{i j}^{\prime} e_{j}
$$

In most cases the latter notation is used. For zinc blende semiconductors the piezoelectric tensor $e_{i j}^{\prime}$ has only one non-zero component, and the polarization becomes

$$
\left(\begin{array}{l}
P_{x} \\
P_{y} \\
P_{z}
\end{array}\right)=\left(\begin{array}{cccccc}
0 & 0 & 0 & e_{14} & 0 & 0 \\
0 & 0 & 0 & 0 & e_{14} & 0 \\
0 & 0 & 0 & 0 & 0 & e_{14}
\end{array}\right)\left(\begin{array}{c}
e_{x x} \\
e_{y y} \\
e_{z z} \\
e_{y z} \\
e_{z x} \\
e_{x y}
\end{array}\right)
$$


Thus the piezoelectricity is generated only by shear strain. The piezoelectric effect is largest for $<111>$ since anions and cations are staked in (111) planes.

The piezoelectric effect plays huge role in optical properties of low dimensional semiconductor structures like quantum dots. This effect will be discussed in later section.

Changes of interatomic distances and the bonding angles under the strain in turn cause modifications in the overlapping integrals and the band structure. The most common way to treat these effects is to use the kp-method as described, e.g., in ref. 9. Here I will point out some qualitative effects of strain on the band structure.

Under hydrostatic pressure only interatomic distances are changed, but the bonding angles remain the same. This results in increased values of the overlapping integrals, and the bandgap is widened as it was discussed in the previous section.

The shear strain has different effects on bands with different symmetries. For the $s$ like $\mathrm{CB}$, only hydrostatic pressure can shift the band but not the shear strain. However the shear strain alters bonding angles of the $p$-like bonds. Under biaxial strain, all four bonds rotate towards or away from each other in the $x-y$ plane depending on whether the strain is tensile or compressive. For a biaxial tension, all four bonds rotate towards the $x-y$ plane resulting in an increased contribution of the $p_{x}$ and $p_{y}$ orbitals and a decreased contribution of the $p_{z}$ orbitals to the hole states. Along [001] $\mid z$, this results in bringing the LH band to the top of VB. However in the [100] I $\mid x$ and [010] I $y$ directions the LH band is pushed down since it is composed mainly of the $p_{x}$ and $p_{y}$ states, and the $\mathrm{HH}$ band becomes the topmost VB. In fact under such conditions there are no "pure" $\mathrm{HH}$ and $\mathrm{LH}$ bands, but rather $\mathrm{LH}$-like or $\mathrm{HH}$-like states. 


\section{Chapter 2 Phonon properties.}

\subsection{Lattice dynamics in harmonic approximation.}

\subsubsection{Equation of motion of oscillating 3D lattice in harmonic approximation.}

We again start with addressing position of each atom in a primitive cell in the way we did in (1.1). Let us assume that each primitive cell contains $l=1 \ldots r$ atoms. We denote the equilibrium position of an $l^{\text {th }}$ atom in $\kappa^{\text {th }}$ cell as $\mathbf{x}\left(\begin{array}{l}l \\ \kappa\end{array}\right)$. Due to thermal fluctuations each atom with mass $M_{\kappa}$ experience shift from the equilibrium positions by value of $\mathbf{u}\left(\begin{array}{l}l \\ \kappa\end{array}\right)$ with $u_{\alpha}\left(\begin{array}{l}l \\ \kappa\end{array}\right) \alpha$-component $(\alpha=x, y, z)$. Let assume that the full potential energy $\Phi$ of the atoms is a function of individual atomic displacements. $\Phi$ can be expanded in Taylor series over the individual atomic displacements as:

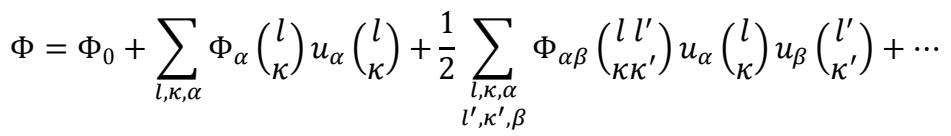

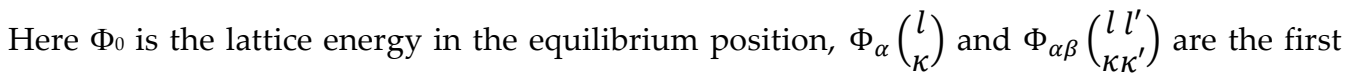
and the second derivatives of $\Phi$ evaluated in the equilibrium. The expansion limited to quadratic terms is known as harmonic approximation. This means that the atoms oscillate around their equilibrium positions corresponding to the minimal potential energy of the crystal. The linear term in the above expansion corresponds to the restoring force acting on the atom. Anharmonic effects are included into the higher order expansions and will not be considered in this thesis.

The Hamiltonian becomes then

$$
H=\Phi_{0}+\frac{1}{2} \sum_{l, \kappa, \alpha} M_{\kappa} u_{\alpha}^{2}\left(\begin{array}{l}
l \\
\kappa
\end{array}\right)+\frac{1}{2} \sum_{\substack{l, k, \alpha \\
l, \kappa^{\prime}, \beta}} \Phi_{\alpha \beta}\left(\begin{array}{l}
l l^{\prime} \\
\kappa \kappa^{\prime}
\end{array}\right) u_{\alpha}\left(\begin{array}{l}
l \\
\kappa
\end{array}\right) u_{\beta}\left(\begin{array}{c}
l^{\prime} \\
\kappa^{\prime}
\end{array}\right)
$$

The equations of motion are given by:

$$
M_{\kappa} \ddot{u}_{\alpha}\left(\begin{array}{l}
l \\
\kappa
\end{array}\right)=-\sum_{l, \kappa^{\prime}, \beta} \Phi_{\alpha \beta}\left(\begin{array}{c}
l l^{\prime} \\
\kappa \kappa^{\prime}
\end{array}\right) u_{\beta}\left(\begin{array}{c}
l^{\prime} \\
\kappa^{\prime}
\end{array}\right)
$$

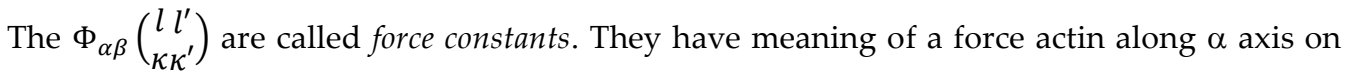
atom in $\mathbf{x}\left(\begin{array}{l}l \\ \kappa\end{array}\right)$ position when the atom in $\mathbf{x}\left(\begin{array}{l}l^{\prime} \\ \kappa^{\prime}\end{array}\right)$ positon is displaced along $\beta$ by unity distance. Due to translational symmetry of the lattice the force constants do not depend on $l, l^{\prime}$ separately but rather on their difference $l-l^{\prime}$. The equations of motion form infinite system of equations. 
So far we were considering the infinite crystal. It is rather easy to treat lattice dynamics in the infinite crystal. However the values associated with the whole crystal, like kinetic or potential energy become infinitely large. In addition to that the range of possible values of the wave vector $\mathbf{k}$ is not defined. Obviosity this range is defined by the boundary conditions. It appears that results of calculations in the harmonic approximation do not really depend on exact selection of the boundary conditions due to negligible contribution of near surface regions with respect to the bulk volume [12].

Thus the simplest boundary conditions are so called cyclic boundary conditions, or Born-Carman conditions. The crystal is assumed to be formed from "macrocrystals" containing $L \times L \times$ $L=N$ primitive cells. These "macrocrystals" are parallelepipeds with edges defined by $L \mathbf{a}_{1}, L \mathbf{a}_{2}, L \mathbf{a}_{3}$. The cyclic boundary conditions require:

$$
u\left(\begin{array}{c}
l+L \\
\kappa
\end{array}\right)=u\left(\begin{array}{l}
l \\
\kappa
\end{array}\right)
$$

The boundary conditions restricts possible values of $\mathbf{k}$ wave vector to:

$$
\mathbf{k}=\frac{1}{L}\left(h_{1} \mathbf{b}_{1}+h_{2} \mathbf{b}_{2}+h_{3} \mathbf{b}_{3}\right)
$$

Here $h_{\mathrm{i}}$ are integer numbers $h_{i}=1 \ldots L, \mathbf{b}_{\mathrm{i}}$ are vectors of the reciprocal lattice. If we look for the solutions in the form of time independent amplitudes:

$$
u_{\alpha}\left(\begin{array}{l}
l \\
\kappa
\end{array}\right)=M_{\kappa}^{-1 / 2} v_{\alpha}\left(\begin{array}{l}
l \\
\kappa
\end{array}\right) e^{-i \omega t}
$$

then the infinite system of equations (2.3) becomes finite system of linear equations

$$
\omega^{2} v_{\alpha}\left(\begin{array}{l}
l \\
\kappa
\end{array}\right)=\sum_{l,, \kappa, \beta}\left(M_{\kappa} M_{\kappa^{\prime}}\right)^{-1 / 2} \Phi_{\alpha \beta}\left(\begin{array}{l}
l l^{\prime} \\
\kappa \kappa^{\prime}
\end{array}\right) v_{\beta}\left(\begin{array}{l}
l^{\prime} \\
\kappa^{\prime}
\end{array}\right)
$$

All physically different solutions of (2.7) are contained within single primitive reciprocal lattice [12]. Thus there are $L^{3}=N$ different values of $\mathbf{k}$. This system has non-trivial solutions when:

$$
\left|\mathrm{D}_{\alpha \beta}\left(\begin{array}{l}
l l^{\prime} \\
\kappa \kappa^{\prime}
\end{array}\right)-\omega^{2}(\mathbf{k}) \delta_{\alpha \beta} \delta_{l l^{\prime}} \delta_{\kappa \kappa^{\prime}}\right|=0
$$

The matrix elements of $\mathrm{D}_{\alpha \beta}\left(\begin{array}{l}l l^{\prime} \\ \kappa \kappa^{\prime}\end{array}\right)$ are deduced from $\left(M_{\kappa} M_{\kappa^{\prime}}\right)^{-1 / 2} \Phi\left(\begin{array}{l}l l^{\prime} \\ \kappa \kappa^{\prime}\end{array}\right)$ by grouping indexes $(\alpha, \kappa, l)$ and $\left(\beta, \kappa^{\prime}, l^{\prime}\right)$. The matrix $\mathrm{D}_{\alpha \beta}$ is called dynamical matrix of the crystal. The equation (2.8) is of $3 r$ order concerning $\omega^{2}$, and for each $\mathbf{k}$ it has $3 r$ solutions which we will denote as $\omega_{j}^{2}(\mathbf{k})$, where $j=1 \ldots 3 r$. Due to definition of $D_{\alpha \beta}$ it can be shown that $\omega_{j}^{2}(\mathbf{k})$ and $\omega_{j}(\mathbf{k})$ are real meaning that the corresponding atomic oscillations are not damped. The $3 \mathrm{r} \omega_{j}^{2}(\mathbf{k})$ functions can be viewed as different branches of multivalued function $\omega^{2}(\mathbf{k})$ [12]. The relation

$$
\omega=\omega_{j}(\mathbf{k}), j=1 \ldots 3 r
$$


is called dispersion relation. In general case the dispersion is obtained only numerically. However some conclusions about possible atomic vibrations can be made without exact knowing the dispersion or without any model system such as diatomic linear chain. Values $\omega_{j}^{2}(\mathbf{k})$ are eigenvalues of $D_{\alpha \beta}$. For each of $3 r$ values of $\omega_{j}^{2}(\mathbf{k})$ with a fixed $\mathbf{k}$ there is a vector $e\left(\left.\kappa\right|_{j} ^{\mathbf{k}}\right)$ which is eigenvector of $D_{\alpha \beta}$ and thus satisfies system (2.7). These vectors are atomic oscillations described by system (2.7). Three of the $3 r$ eigenvectors vanish as $\mathbf{k} \rightarrow 0$, one for each space direction $(\mathrm{x}, \mathrm{y}, \mathrm{z})$. These oscillations are called acoustic modes. Atoms in these oscillations in limit of vanishing $\mathbf{k}$ oscillate in phase with the same amplitude, what is typical for propagation of sound waves in an elastic medium. The rest of $3 r-3$ oscillations are called optical modes, and the atoms oscillate in antiphase while the center of mass is still. In case of nonpolar crystals with cubic symmetry all optical modes are degenerate at $\mathbf{k}=\mathbf{0}$ [12].

\subsubsection{Normal coordinates and symmetry of lattice oscillations.}

The crystal Hamiltonian (2.2) is a quadratic form made of atomic displacements and momentum components. Therefore it can be diagonalized. Let expand the atomic displacements $u_{\alpha}\left(\begin{array}{l}l \\ \kappa\end{array}\right)$ in series of plane waves:

$$
u_{\alpha}\left(\begin{array}{l}
l \\
\kappa
\end{array}\right)=\frac{1}{\sqrt{N M_{\kappa}}} \sum_{\mathbf{k}, j} e_{\alpha}\left(\kappa \mid \begin{array}{l}
\mathbf{k} \\
j
\end{array}\right) Q\left(\begin{array}{c}
\mathbf{k} \\
j
\end{array}\right) e^{2 \pi i \mathbf{k} \cdot \mathbf{x}(l)}
$$

Then the equation of motion transforms to simple form of a harmonic oscillator in coordinates $Q\left(\begin{array}{c}\mathbf{k} \\ j\end{array}\right)$ :

$$
\ddot{Q}\left(\begin{array}{c}
\mathbf{k} \\
j
\end{array}\right)+\omega_{j}^{2}(\mathbf{k}) Q\left(\begin{array}{c}
\mathbf{k} \\
j
\end{array}\right)=0
$$

We can see that the expansion coefficients $Q\left(\begin{array}{l}\mathbf{k} \\ j\end{array}\right)$ of $u_{\alpha}\left(\begin{array}{l}l \\ \kappa\end{array}\right)$ in (2.10) play role of coordinates and called normal coordinates. From the equation above it follows that each of normal coordinates is a simple periodic function of time with frequency $\omega_{j}^{2}(\mathbf{k})$. Each of these coordinates describes one if independent oscillations of the crystal with the given frequency, i.e. an elastic wave of oscillations which propagates through the crystal. These oscillations are called normal oscillations. In each normal oscillation all the atoms oscillate with the same frequency and phase. There are as much normal coordinates as there are degrees of freedom of the crystal, i.e. $3 r N$. From the definition of normal coordinates it follows that lattice dynamics of a crystal in general case is a superposition of normal oscillations with weight $e_{\alpha}\left(\left.\kappa\right|_{j} ^{\mathbf{k}}\right) e^{2 \pi i \mathbf{k} \cdot \mathbf{x}(l)}$.

The normal coordinates are useful tool to quantize the lattice vibrations. The equation (2.11) 
can be rewritten in second quantization formalism using creation $a_{j}(\mathbf{k})$ and annihilation $a_{j}^{+}(\mathbf{k})$ operators as:

$$
H=\sum_{\mathbf{k}, j} \hbar \omega_{j}(\mathbf{k})\left(a_{j}^{+}(\mathbf{k}) a_{j}(\mathbf{k})+1 / 2\right)
$$

This is precisely equation of quantum harmonic oscillator. Therefore the lattice vibrations can be viewed as quanta of lattice vibrations in the same way as photons are quanta of the electromagnetic field. These quanta of lattice vibrations are called phonons and represent lattice oscillations of the crystal of particular energy and phase.

The normal coordinates can provide fairly easy way to determine the symmetries of phonon modes at the center of BZ. The normal coordinates are invariant under the symmetry operations $R$ of the crystal space group. The set of coordinates degenerate among themselves indexed $r, r^{\prime}, r^{\prime \prime} \ldots$ forms the basis of an irreducible representation $\chi$ of the crystal's space group. Their number is equal to the dimension of the representation [13].

As we remember at the $\Gamma$ point the group of wave vector coincides with the point group of the crystal. Thus the normal coordinates transform as:

$$
Q\left(\begin{array}{l}
\mathbf{0} \\
j
\end{array}\right) \stackrel{R}{\rightarrow} Q^{\prime}\left(\begin{array}{l}
\mathbf{0} \\
j
\end{array}\right)=\sum_{n} D^{(i)}(R)_{n j} Q\left(\begin{array}{l}
\mathbf{0} \\
j
\end{array}\right)
$$

here $\mathrm{D}^{(\mathrm{i})}$ denotes the matrix of irreducible representation of the crystal point group. Thus in order to identify the symmetries of the phonon modes of the lattice, one need to identify character of the representation $\chi$.

The character is calculated in the following way. We choose any primitive cell and count number $U_{R}$ of positions $j=1 \ldots r$ which remain unchanged under the effect of the symmetry operation R. We multiply this number by character of the operation $\mathrm{R}: \xi=U_{R} \operatorname{Tr}\left(D^{(i)}(R)\right)$. Then we use standard decomposition procedure of the reducible representation $\chi$ to irreducible representations $\Gamma^{i} \subset G$ ( $G$ is the crystal point group) [13]. 


\subsubsection{Zone folding of phonons.}

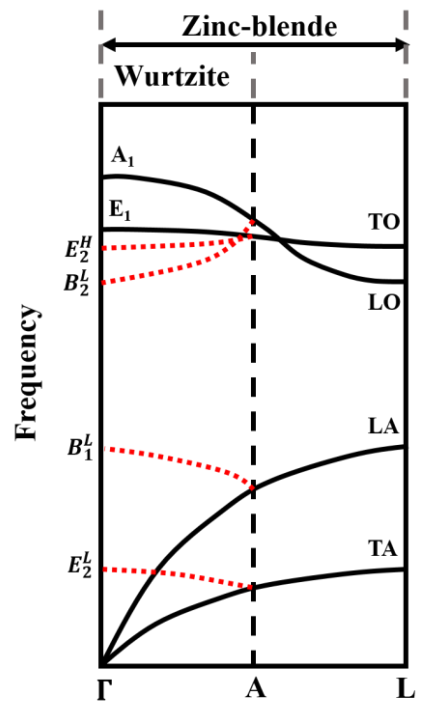

Figure 5. Schematic illustration of the zone folding concept.
Some ZB semiconductors, like GaAs or InP, may crystallize in $\mathrm{WZ}$ phase depending on the crystallization conditions. If the phonon dispersion is known for the ZB structure, the phonon dispersion of $\mathrm{WZ}$ phase can be estimated by the zone folding concept [14], [15].

The structural difference between $\mathrm{ZB}$ and $\mathrm{WZ}$ phase is sequence of atomic layers stacking. The atomic stacking sequence of $\mathrm{ZB}$ phase along [111] can be resented as "ABCABCABC where A-B$\mathrm{C}$ denotes position of the Ga-As pair. The WZ phase stacking is "ABABABAB..." along [0001] [16]. The unit cell length of WZ phase along [0001]

doubles those of $\mathrm{ZB}$ in [111], then the Brillouin zone folding relation $\mathrm{L}(\mathrm{ZB}) \rightarrow \Gamma(\mathrm{WZ})$ holds [17]. The operation of the zone folding is schematically represented on picture below. As result of this procedure new modes appear at $\Gamma$ point. The schematic illustration of the zone folding concept is shown on Figure 5.

\subsubsection{LO-TO phonon splitting.}

The optical phonon branch in fact consists of transverse optical (TO) and longitudinal optical (LO) branches. TO modes correspond to oscillations of atoms in the direction of the wave propagation, while they oscillate orthogonally to the wave propagation in case of the LO mode. In cubic nonpolar crystals these modes are degenerate by symmetry. Indeed, semiconductors like Si or Ge have only one optical phonon branch. However in case of polar semiconductors, like GaAs, the LO and TO branches are split.

The origin of the splitting can be qualitatively understood as follows. The splitting happens due to partially ionic character of chemical bonds in this type of semiconductors. For example, in GaAs Ga atoms have on average positive charge, while As atoms become negatively 
charged. Assume that LO and TO phonons propagate along the [111] direction. Then the positive and negative "ions" are located in separate planes orthogonal to the [111] direction. In the LO mode, these planes of atoms oscillate in the direction of propagation. Thus the distance between charges changes, and the restoring force due to Coulomb interaction appears. The restoring force does not appear in case of transverse oscillations of the atomic planes, i.e. for the TO mode. In another words, the propagation of an LO phonon leads to the appearance of a macroscopic electric field. This electric filed obviously propagates over many primitive cells of the crystal lattice, i.e. it has long-range nature. The electric field changes force constant of the LO phonon and thus lifts the degeneracy of the LO and TO modes. The analogy with a flat-plate capacitor can be useful to understand the picture. Changes in the distance between the capacitor's plates results in variation of its energy, while sliding them with the same distance does not affect the stored energy of the capacitor.

The situation is more complicated in the wurtzite semiconductors. Due to the lowered symmetry, the optical mode at the zone center is split into 2 modes. The first one belongs to the one-dimensional representation of the $\mathrm{C}_{6 \mathrm{v}}$ group ( $\mathrm{A}_{1}$ or $\Gamma_{1}$ in the Koster notations), which transforms as the $z$ component of a vector $(z|| c)$. The second mode belongs to the two-dimensional representation ( $E_{1}$ or $\Gamma_{5}$ in the Koster notations), which transforms as $(x, y)$ components of a vector. This splitting happens due to anisotropy of the force constants. Since most of WZ semiconductors are polar, like $\mathrm{ZnO}$, these modes are split additionally into the $\mathrm{LO}$ and $\mathrm{TO}$ modes [18].

When the phonon propagates parallel or orthogonally to the $c$-axis, it retains its "pure" TO or LO nature of a certain symmetry. When the propagation direction is arbitrary the situation is complicated. When effects of the electrostatic forces dominate over anisotropy of the force constants (which is the case for all real WZ crystals), the TO and LO phonons keep the transverse or orthogonal nature in any arbitrary direction. However they have mixed $A_{1}$ and $E_{1}$ symmetry character, i.e. modes of different symmetry mix [18], [19]. The frequency of phonons then are given by:

$$
\begin{aligned}
& \omega_{L O}^{2}=\omega_{A_{1}(L O)} \cos ^{2}(\theta)+\omega_{E_{1}(L O)} \sin ^{2}(\theta) \\
& \omega_{T O}^{2}=\omega_{A_{1}(T O)} \cos ^{2}(\theta)+\omega_{E_{1}(T O)} \sin ^{2}(\theta)
\end{aligned}
$$

Thus we can see that the LO-TO splitting generally speaking depends on anisotropy of force constants and strength of the long-range electrostatic forces [18]-[20]. Changes in LOTO splitting provide insight in structural changes of the material due to a treatment, like effects of ion implantation in GaAs [21], or effect on N incorporation in GaAs [22]. 


\subsection{Inelastic light scattering.}

\subsubsection{Simple classical treatment.}

Light scattering spectroscopies provide a powerful and non-destructive tool for structural studies of solids. In addition to transmission and absorption of light, some fraction of it may scatter. The scattered photons may be of the same energy as incident ones. Since their energy does not change, this sort of scattering is known as elastic, or Rayleigh scattering. However the photon can transfer (or gain) some of its energy to the medium. In this case its energy becomes less (higher) than that before the scattering event. This sort of scattering is known as inelastic scattering. The process where a photon loses (gain) its energy is known as Stockes (anti-Stockes) scattering. Analyzing the spectrum of the scattered light, one can draw conclusions about excitations of the medium involved in the scattering event and, therefore, gather some information about the structure. In this section we discuss the classical treatment of inelastic light scattering.

Consider an infinite medium with electric susceptibility $\chi$ and assume the incident light to be a sinusoidal plane wave of frequency $\omega_{i}$ and amplitude $\mathbf{F}\left(\mathbf{k}_{i}, \omega_{i}\right)$. This wave will induce sinusoidal polarization of the medium $\mathbf{P}(\mathbf{r}, \mathrm{t})$ :

$$
\mathbf{P}(r, t)=\mathbf{P}\left(\mathbf{k}_{i}, \omega_{i}\right) \cos \left(\mathbf{k}_{i} \mathbf{r}-\omega_{i} t\right)
$$

The induced polarization and the incident light wave are related to each other by:

$$
\mathbf{P}\left(\mathbf{k}_{\mathbf{i}}, \omega_{i}\right)=\chi\left(\mathbf{k}_{\mathbf{i}}, \omega_{i}\right) \mathbf{F}\left(\mathbf{k}_{\mathbf{i}}, \omega_{i}\right)
$$

At finite temperature the lattice oscillations will introduce fluctuations in the electric susceptibility $\chi$. At temperatures well below melting the atomic displacements are small compared to interatomic distances, and we can expand the susceptibility in series over normal coordinates, denoted as $\mathbf{Q}\left(\mathbf{q}, \omega_{p h}\right)$ for a phonon with wavevector $\mathbf{q}$ and frequency $\omega_{p h}$ :

$$
\chi\left(\mathbf{k}_{i}, \omega_{i}, \mathbf{Q}\right)=\chi_{0}+\left.\frac{\partial \chi}{\partial \mathbf{Q}}\right|_{0} \mathbf{Q}+\cdots
$$

Here $\chi_{0}$ denotes electric susceptibility without fluctuations. The second term represents oscillating susceptibility caused by the thermal fluctuations due to lattice vibrations. Therefore the induced polarization now can be divided in "stationary" (i.e. without thermal oscillations) $\mathbf{P}_{0}(\mathbf{r}, t)$ and "oscillating parts" $\mathbf{P}_{\text {ind }}(\mathbf{r}, t, \mathbf{Q})$ induced by propagation of a phonon:

$$
\mathbf{P}(r, t)=\mathbf{P}_{0}(\mathbf{r}, t)+\mathbf{P}_{\text {ind }}(\mathbf{r}, t, \mathbf{Q})
$$

The stationary part vibrates in phase with the incident radiation, and the oscillating part is: 


$$
\begin{aligned}
\mathbf{P}_{\text {ind }}(\mathbf{r}, t, \mathbf{U})= & \left.\frac{\partial \chi}{\partial \mathbf{Q}}\right|_{0} \mathbf{Q}\left(\mathbf{q}, \omega_{p h}\right) \mathbf{F}\left(\mathbf{k}_{\mathbf{i}}, \omega_{i}\right) \\
& \times\left\{\cos \left[\left(\mathbf{k}_{i}+\mathbf{q}\right) \cdot \mathbf{r}-\left(\omega_{i}+\omega_{p h}\right) t\right]\right. \\
& \left.+\cos \left[\left(\mathbf{k}_{i}-\mathbf{q}\right) \cdot \mathbf{r}-\left(\omega_{i}-\omega_{p h}\right) t\right]\right\}
\end{aligned}
$$

We see that the fluctuating polarization part consists of two harmonic waves. One represents Stokes wave with the frequency $\omega_{S}=\omega_{i}-\omega_{p h}$ and the wave vector $\mathbf{k}_{S}=\mathbf{k}_{i}-\mathbf{q}$, so the photon transfers its energy to the crystal. Another one represents anti-Stokes wave with the frequency $\omega_{A S}=\omega_{i}+\omega_{p h}$ and the wave vector $\mathbf{k}_{A S}=\mathbf{k}_{i}+\mathbf{q}$, and the photon gains energy from the solid. The radiation emitted by these polarization waves is known as Stokes and anti-Stokes shifts respectively. The anti-Stokes process will only happen if the crystal is in an excited state. Therefore the intensity of the anti-Stokes process is much weaker than that of the Stokes one and depends on temperature. The frequency difference between the incident and scattered waves is known as the Raman shift.

During the scattering event momentum is preserved:

$$
\mathbf{k}_{\text {incident }}^{\text {photon }}=\mathbf{q}^{\text {phonon }}+\mathbf{k}_{\text {scattered }}^{\text {phton }}
$$

Since wave vector of a photon of visible spectrum is of order $10^{6} \mathrm{~cm}^{-1}$ what is about 1/1000 of the size of Brillouin zone, only zone-center phonons participate in the scattering process. Therefore one-phonon light scattering probes only those phonons of $\mathbf{q}^{\text {phonon }} \approx 0$. Usually the phonon wave vector in such experiments is assumed to be 0 in theoretical calculations. In the following we will restrict ourselves to the Stokes scattering process.

Let us denote polarization of the scattered light as $\mathbf{e}_{\mathbf{s}}$, and those of the incident wave as $\mathbf{e}_{\mathbf{i}}$. Then the intensity of scattered light $\mathrm{I}_{\mathrm{s}}$ is proportional to [19]:

$$
I_{s} \sim\left|\mathbf{e}_{i}\left(\left.\frac{\partial \chi}{\partial Q}\right|_{0} \mathbf{Q}\left(\omega_{p h}\right)\right) \mathbf{e}_{s}\right|
$$

We denote $R_{S}=\left.\frac{\partial \chi}{\partial \mathbf{Q}}\right|_{0} \cdot \frac{\mathbf{Q}}{|\mathbf{Q}|}$. This entity is known as Raman tensor and its non-zero elements can be identified by symmetry considerations, see [19] and references therein. Then the expression above can be rewritten in the most common way:

$$
I_{S} \sim\left|\mathbf{e}_{\mathbf{s}} R_{s} \mathbf{e}_{i}\right|^{2}
$$

From a microscopic point of view, the Raman scattering is a three-step process: 1$)$ excitation of an electron from the ground state $|g\rangle$ to the intermediate electron-hole $(e-h)$ pair state $\left|i_{1}\right\rangle$; 2 ) scattering of the $e-h$ pair into another state $\left|i_{2}\right\rangle$ with emission (Stokes process) or absorption (anti-Stokes process) phonon; 3 ) recombination of the $e-h$ pair in $\left|i_{2}\right\rangle$ into the ground state with emission of a photon whose energy is then detected. In the case of non-resonant Raman 
scattering, i.e. far away from resonances, the $e-h$ pair is virtual one. The detailed discussion can be found in numerous books edited by M. Cardona.

\subsubsection{Selection rules.}

The Raman scattering rules are already contained in the Raman tensor. Thus, knowing it, one can identify which modes participate in a certain scattering geometry and determine their polarization. The derivation of the Raman tensor is usually based on symmetry considerations and can be found elsewhere [19]. However, even without detailed knowledge of the Raman tensor one can deduce what modes will participate in the Raman scattering.

The entity $P_{\alpha \beta}$

$$
P_{\alpha \beta}=\sum_{l, \kappa, \gamma} P_{\alpha \beta, \gamma} u_{\gamma}\left(\begin{array}{l}
l \\
\kappa
\end{array}\right)
$$

where:

$$
P_{\alpha \beta, \gamma}=\partial \chi_{\alpha \beta} / \partial u_{\gamma}\left(\begin{array}{l}
l \\
\kappa
\end{array}\right)
$$

is sometimes called polarizability. For the $\mathbf{k}=0$ the polarizability becomes proportional to the Raman tensor and can be expressed thought normal coordinates in the following way [23]:

$$
P_{\alpha \beta}=\sqrt{N} \cdot \sum_{j} P_{\alpha \beta}(j, \mathbf{0}) Q\left(\begin{array}{l}
\mathbf{0} \\
j
\end{array}\right)
$$

where:

$$
P_{\alpha \beta}(j, \mathbf{0})=\sum_{\gamma, \kappa} P_{\alpha \beta, \gamma} e\left(\kappa \mid \begin{array}{l}
\mathbf{0} \\
j
\end{array}\right) \cdot 1 / \sqrt{ } m_{\kappa}
$$

Under symmetry operations of the crystal point group normal coordinates $Q\left(\begin{array}{l}\mathbf{0} \\ j\end{array}\right)$ are invariant. Thus the polarizability tensor is invariant under these transformation. From the other hand, it should transform as tensor under this symmetry operation $R$ as:

$$
P_{\alpha \beta}^{\prime}=\sum_{\gamma \delta} R_{\alpha \gamma} R_{\beta \delta} P_{\gamma \delta}
$$

where $R_{i j}$ means matrix representation of the symmetry operation $R$. If the reduced form of the entity

$$
R_{\alpha \beta, \epsilon \eta}=\left\{\begin{array}{c}
R_{\alpha \epsilon} R_{\beta \eta}+R_{\beta \epsilon} R_{\alpha \eta}, \text { if } \alpha \neq \beta \\
R_{\alpha \epsilon} R_{\alpha \eta}, \text { if } \alpha=\beta
\end{array}\right.
$$

contains irreducible representation $\Gamma^{i}$, and a phonon mode transforms as $\Gamma^{i}$, then the phonon of $\Gamma^{i}$ symmetry participates in the Raman scattering process. The modes, which participate in the scattering process are called Raman active mode. The modes which do not participate in 
both IR absorption and the Raman scattering are called silent modes.

\subsubsection{Electron-optical phonon interaction.}

Long wavelength optical phonons involve relative displacements of atoms within single primitive cell. These displacements produce deformations of the primitive cell. In nonpolar semiconductors these deformations cause changes in the bond lengths and/or the bond angles. This electron-optical phonon interaction is known as deformation-potential electron-(optical) phonon interaction. This interaction does not depend on the phonon wave vector $\mathbf{k}$ and therefore it is short range interaction. The quantitate characteristic of these changes are phonon deformation potentials (PDP) defined as the change in the spring constant $K_{i k}$ due to applied strain:

$$
K^{(1)}=\frac{\partial K_{i k}}{\partial \epsilon_{l m}} ; i, k, l, m=x, y, z
$$

In cubic crystals there are only 3 independent components of the tensor $K^{(1)}$ [24]:

$$
\begin{gathered}
K_{1111}^{1}=K_{2222}^{1}=K_{3333}^{1}=\mu p \\
K_{1122}^{1}=K_{2233}^{1}=K_{1133}^{1}=\mu q \\
K_{1212}^{1}=K_{2323}^{1}=\mu r
\end{gathered}
$$

where $\mu$ is the reduced mass. The deformation potentials are rather difficult to calculate theoretically [25] [26].

In polar semiconductors the long-wavelength LO phonon generates macroscopic electric field. Electrons in the crystal can couple to the field. This electron-LO phonon interaction is known as Fröhlich interaction. Contrary to the deformation potentials, the Fröhlich interaction can be fairly easy calculated, which appears to be a long-range interaction since the strength depends inversely on the phonon wave vector q. Furthermore, the Raman scattering intensity is proportional to magnitude of $\mathbf{q}$. For impurity-mediated scattering, when the electron in the intermediate state is localized on the impurity atom, the $\mathbf{q} \approx 0$ condition is broken, and the scattering amplitude increases significantly [27].

\subsection{Strain and alloying effects on Raman spectrum.}

\subsubsection{Strain-induced effects.}

In the absence of stress the optical phonons at zone center are triply degenerate in diamond-like and ZB crystals. The application of uniaxial strain lifts the degeneracy of the phonon modes [24], [28].

For uniaxial stress along [001] and [111] directions the threefold degenerate phonon modes are split into a singlet $\left(\Omega_{\mathrm{s}}\right)$ with eigenvector parallel to the strain axis and a doublet $\left(\Omega_{\mathrm{d}}\right)$ with 
eigenvector orthogonal to the strain axis. The frequency of the optical modes are given then as [24]:

$$
\begin{aligned}
& \Omega_{s}=\omega_{0}+\Delta \Omega_{H}+\frac{2}{3} \Delta \Omega \\
& \Omega_{d}=\omega_{0}+\Delta \Omega_{H}-\frac{1}{3} \Delta \Omega
\end{aligned}
$$

Here $\omega_{0}$ is the frequency without strain, $\Omega_{\mathrm{H}}$ is a shift due to presence of the hydrostatic component in the strain. These quantities can be expressed through phonon deformation potentials and the applied stress $\mathbf{X}$ as following [24]:

$$
\begin{gathered}
\Delta \Omega_{h}=\left(X / 6 \omega_{0}\right)(p+2 q)\left(S_{11}+2 S_{12}\right) \\
\Delta \Omega=\Omega_{s}-\Omega_{d}= \begin{cases}=\Delta \Omega^{[001]}=\left(X / 2 \omega_{0}\right)(p-q)\left(S_{11}-S_{12}\right), & \mathbf{X} \|[001] \\
=\Delta \Omega^{[111]}=\left(X / 2 \omega_{0}\right) r S_{44} & \mathbf{X ~ \| ~ [ 1 1 1 ] ~}\end{cases}
\end{gathered}
$$

Application of stress along [110] splits the triply degenerate modes into 3 components [24]:

$$
\begin{gathered}
\Omega_{1}=\omega_{0}+\Delta \Omega_{H}-\frac{1}{3} \Delta \Omega^{[001]} \\
\Omega_{2}=\omega_{0}+\Delta \Omega_{H}+\frac{1}{6} \Delta \Omega^{[001]}+\frac{1}{2} \Delta \Omega^{[111]} \\
\Omega_{2}=\omega_{0}+\Delta \Omega_{H}+\frac{1}{6} \Delta \Omega^{[001]}-\frac{1}{2} \Delta \Omega^{[111]}
\end{gathered}
$$

Qualitatively application of stress changes bond lengths and/or bond angles. This results in changes of force constants. Therefore the tensile strain will result in decreasing of phonon frequencies. This situation occurs, for example, when a thin film of a material with smaller lattice constant is grown on top of a material with larger lattice constant. Example of such system can be a thin film of GaNAs grown on top of GaAs or a shell of GaNAs grown on $\mathrm{GaAs}$ core in $\mathrm{GaAs} / \mathrm{GaNAs}$ nanowire core/shell strucutres. In the former case strong shift was observed as more $\mathrm{N}$ was incorporated [29].

\subsubsection{Alloying.}

The presence of a small concentration of substitutional impurity might be regarded as ultra-dilute regime of alloying. This results in changes of force constants and may lead to breaking of the translational symmetry of the host lattice. Therefore the frequencies of normal modes and associated eigenvectors are altered. Basically there are two sorts of vibrational modes created by the substitutional impurity: localized and resonance modes. A localized mode has its frequency in the range of the forbidden normal mode frequencies of the perfect host crystal. Its vibrational amplitude is strongly localized on the impurity atom and fats decays with increased distance to the atom. A localized mode named local mode if its frequency is higher than the maximum vibrational frequency of the perfect host lattice. A localized mode 
named gap mode if its frequency belongs to range between the optical and the acoustic modes of the host. A resonance mode is defined as mode whose frequency belongs to the range of the allowed frequencies of normal modes of the host lattice. It depends on the mass defect parameter whether the mode is localized or resonance [30].

One can identify three classes of mixed crystals of the type $A_{x} B_{1-x} C$ in terms of their optical phonon modes behavior.

- One-mode behavior. In this cases zone-center optical modes vary continuously with the concentration $\mathrm{x}$. They vary from those of $\mathrm{AB}$ material to modes of $\mathrm{BC}$ material. All the frequencies appear approximately with the same strength.

- Two-mode behavior. At a given concentration $x$ there are two sets of optical modes with frequencies characteristic of each end alloy AB and BC. Their strengths are approximately proportional to the $x$.

- Mixed mode behavior. Two-mode behavior is observed in some range of concentrations, and for the rest of $x$ there is one-mode behavior.

The most successful model of mixed crystals is so called modified random element isodisplacement (MREI) introduced by [31], [32]. The theory makes following assumptions:

1. Isodusplacement. The ions are considered to oscillate with the same phase and amplitude. This assumption is valid for the zone center phonons.

2. Randomness. $\mathrm{B}$ and $\mathrm{C}$ ions are assumed to be randomly distributed on the anion sub lattice. Therefore each atom is exposed to forces produced by a statistical average of its neighbors. In zinc blende crystals each $A$ is surrounded by $4 x$ atoms $C$ and $4(1-x)$ atoms of $B$. Both B and C then have 12(1-x) next-nearest neighbors of B and $12 x$ nextnearest neighbors of $\mathrm{C}$.

The theory predicts that alloys which obey $m_{B}<\mu_{A C}$ exhibit two-mode behavior and opposite is true for one-mode behavior. In another words an alloy $\mathrm{A}_{x} \mathrm{~B}_{1-x} \mathrm{C}$ in order to demonstrate two-mode behavior must have one substituting element whose mass is smaller than reduced mass of the compound formed by the other 2 elements. Figure 6 schematically illustrates the mode behavior. 


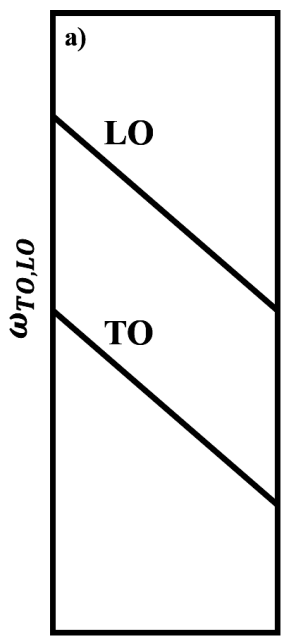

AB

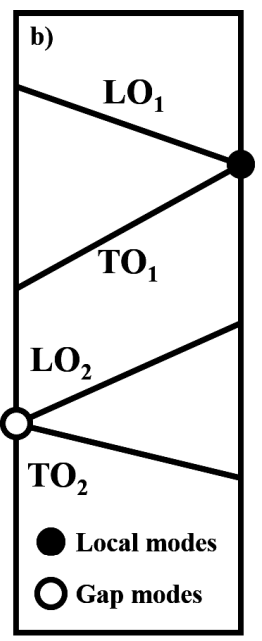

$\mathbf{B C} \mathbf{A B}$
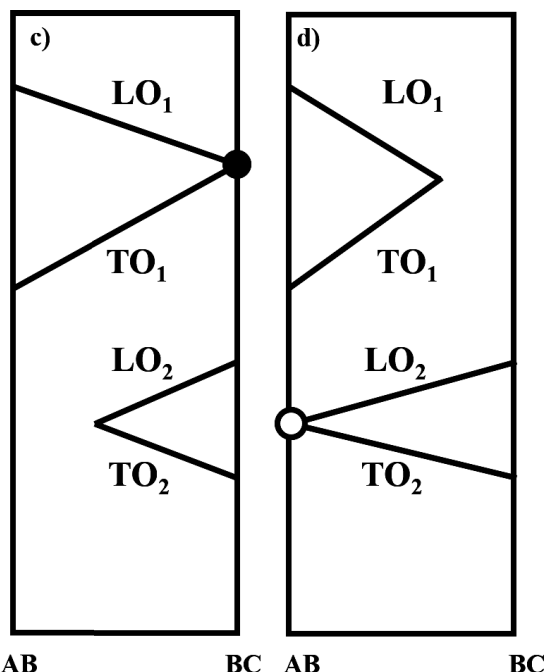

\section{Composition}

Figure 6. Schematic illustration of four different types of long wavelength phonon modes behavior in a ternary alloy $A_{x} B_{1-x} C:$ a)one mode; b) two mode; c) mixed mode (1); d) mixed mode (2)

\subsection{Surface optical modes.}

Surface optical (SO) phonons propagate in the near to surface region, i.e. the atomic displacements are confined near to the surface. The amplitudes of these phonons decay exponentially into the depth of the material [33]. The electric field associated with LO phonons will be different near the surface, and thus the frequency of such phonons will differ from the bulk value. The frequency of a SO phonon can be calculated in framework of dielectric continuum model by solving the Laplace equation for a given boundary conditions. The SO modes are often observed in NWs and were theoretically studied for NWs of various geometries in [34], [35].

Let consider a cylinder of radius $a$ and the outer medium with dielectric constant $\epsilon_{d}$. The dielectric constant tensor (real part) is then can be written for WZ or ZB structures as:

$$
\epsilon(\omega)=\epsilon_{\perp}(\omega)\left(\begin{array}{ccc}
1 & 0 & 0 \\
0 & 1 & 0 \\
0 & 0 & g(\omega)
\end{array}\right)
$$

In WZ crystals $g(\omega)=\epsilon_{\|}(\omega) / \epsilon_{\perp}(\omega)$. The components of $\epsilon(\omega)$ are given by Loudon's relations for uniaxial crystal [19]:

$$
\epsilon_{\perp}(\omega)=\epsilon_{\perp}^{\infty}\left(\omega^{2}-\omega_{\perp, L O}^{2}\right) /\left(\omega^{2}-\omega_{\perp, T O}^{2}\right)
$$




$$
\epsilon_{\|}(\omega)=\epsilon_{\|}^{\infty}\left(\omega^{2}-\omega_{\|, L O}^{2}\right) /\left(\omega^{2}-\omega_{\|, T O}^{2}\right)
$$

here $\epsilon_{\perp}^{\infty}$ and $\epsilon_{\|}^{\infty}$ are the high frequency dielectric constants perpendicular and parallel to the $c$-axis, respectively; $\omega_{\perp, L O}^{2}, \omega_{\perp, T O}^{2}$ are the zone center frequencies of $E_{1}(\mathrm{LO})$ and $E_{1}(\mathrm{TO}), \omega_{\|, L O}^{2}$, $\omega_{\|, T O}^{2}$ are the zone center frequencies of $A_{1}(\mathrm{LO})$ and $A_{1}(\mathrm{TO})$ respectively of the WZ crystals; $\omega$ is the SO phonon frequency. In case of cubic crystals $g(\omega)=1$ and the components of $\epsilon(\omega)$ are given by well-known Lyddane-Sachs-Teller relation.

In case of cylindrical wire with axially symmetrical phonon potential of the polar optical modes the SO modes follow the dispersion [36]:

where:

$$
\epsilon_{\perp}(\omega) \sqrt{g(\omega)}+\epsilon_{D} \frac{I_{0}\left(i q_{r} a\right) K_{1}\left(q_{z} a\right)}{I_{1}\left(i q_{r} a\right) K_{0}\left(q_{z} a\right)}=0
$$

$$
\epsilon_{\perp}(\omega) q_{r}^{2}+\epsilon_{\|}(\omega) q_{z}^{2}=0
$$

here $I_{\mathrm{i}}$ and $K_{\mathrm{j}}$ are the Bessel functions of the first and the second kind respectively, $\epsilon_{D}$ is dielectric constant of the surrounding medium, and the $q_{\mathrm{r}}$ and $q_{\mathrm{z}}$ are the radial and axial components of the wavevectors of the SO phonon linked to each other by second formula of (2.34) respectively. The SO modes are solutions of the first equation of (2.34), and their frequencies are always in between those of TO and LO modes. The position of the SO mode depends on the dielectric constant of the surrounding medium [36]-[38]. The dispersion is also nanowire shape-sensitive [34]. 


\section{Chapter 3 Exciton in semiconductors.}

\subsection{Free exciton in bulk.}

In a ground state of a crystal a valence band is fully occupied and the conduction band is empty. However under certain perturbation, such as electro-magnetic field, an electron can be promoted from VB to CB. This results in appearance of a free hole and a free electron which can experience mutual Coulomb attraction. Hence they may form a two-particle system called exciton. The exciton represents an excited state of the crystal. The radius of such correlated pair may extend over several primitive cells, and this exciton is called Wannier exciton. The Wannier excitons are mostly found in semiconductors. However the pair may be constructed from ground and excited states of the same host lattice atom. Such exciton is called Frenkel exciton. The latter is typical case for alkali halides crystals. In the following we will focus on case of Wannier exciton.

Let us denote as $\boldsymbol{\beta}$ the electron-hole separation in real space and introduce the wavevector $\mathbf{K}=\mathbf{k}_{e}-\mathbf{k}_{h}$, where $\mathbf{k}_{e}\left(\mathbf{k}_{h}\right)$ is the electron (hole) wave vector. The wave function of the exciton should depend explicitly on $\mathbf{K}$ since the total momentum of the electron-hole pair is a good quantum number. From the other hand the exciton energy should depend on the electronhole separation. Wannier suggested that the exciton wave function can be formed as series of many-electron wave functions of the excited state:

$$
\begin{gathered}
\Psi_{m n}(\mathbf{K}, \boldsymbol{\beta})=N^{-\frac{1}{2}} \sum_{\mathbf{k}} e^{-i \boldsymbol{\beta} \cdot \mathbf{k}} \Phi_{m n}(\mathbf{k}-\mathbf{K}, \mathbf{k}) \\
=N^{-\frac{1}{2}} \sum_{\mathbf{R}} e^{i \boldsymbol{\beta} \cdot \mathbf{R}} \Phi_{m n}(\mathbf{R}, \mathbf{R}+\boldsymbol{\beta})
\end{gathered}
$$

Here $\Phi_{m n}(\mathbf{k}-\mathbf{K}, \mathbf{k})$ and $\Phi_{m n}(\mathbf{R}, \mathbf{R}+\boldsymbol{\beta})$ are many-body wavefucntions of the excited state of the crystal with hole and electron in particular bands $m$ and $n$ respectively, $\mathrm{N}$ number of the particles under consideration and $\mathbf{R}$ is lattice vector. These functions are constructed as antisymmetric product of single-particle wave functions of electron and hole with the anti-symmetrizing parameter $\mathcal{A}$ of the form:

$$
\mathcal{A}=(N !)^{-\frac{1}{2}} \sum_{P}(-1)^{p} P
$$

Here $P$ is one of the $N$ ! possible permutations with parity $p$. The single electron wavefunctions can be taken as Bloch of Wannier functions which are Fourier transform of each other.

Then one considers the matrix elements $H_{m n, m^{\prime} n^{\prime} ; \mathbf{K}, \beta, \beta^{\prime}}=\left\langle\Phi_{m n}(\mathbf{K}, \boldsymbol{\beta})\left|H_{0}\right| \Phi_{m \prime n^{\prime}}\left(\mathbf{K}, \boldsymbol{\beta}^{\prime}\right)\right\rangle$ where $H_{0}$ is Hamiltonian of the exciton 


$$
H_{0}=-\sum_{i} \frac{\hbar^{2} \nabla_{i}^{2}}{2 m}+\sum_{i} V\left(\mathbf{r}_{i}\right)+\sum_{i<j} \frac{e^{2}}{\epsilon\left|\mathbf{r}_{i}-\mathbf{r}_{j}\right|}
$$

Here the first term describes the kinetic energy of electrons, the second describes effective potential energy of an electron at position $\mathbf{r}_{i}$ with nuclei, the third describes electron-electron interaction in medium with dielectric constant $\varepsilon$. In order to obtain the energy spectrum of an exciton one needs to diagonalize the Hamiltonian $H_{0}$. This can be done by unitary transformation of form:

$$
\Psi_{m n \mathbf{K}}=\sum_{\beta} U_{m n \mathbf{K}}(\boldsymbol{\beta}) \Phi_{m n}(\mathbf{K}, \boldsymbol{\beta})
$$

The transformation coefficients are the solutions of the equations:

$$
\sum_{\boldsymbol{\beta} \prime}\left\langle m n \mathbf{K} \boldsymbol{\beta}\left|H_{0}\right| m n \mathbf{K} \boldsymbol{\beta}^{\prime}\right\rangle=E U_{m n \mathbf{K}}(\boldsymbol{\beta})
$$

They are a subject of the secular equation for the eigenvalues $E$ :

$$
\operatorname{det}\left|\left\langle m n \mathbf{K} \boldsymbol{\beta}\left|H_{0}\right| m n \mathbf{K} \boldsymbol{\beta}^{\prime}\right\rangle-E \delta_{\boldsymbol{\beta} \boldsymbol{\beta} \prime}\right|=0
$$

We used a short notation $|m n \mathbf{K} \boldsymbol{\beta}\rangle \equiv \Phi_{m n}(\mathbf{K}, \boldsymbol{\beta})$, etc. These transformation coefficients can be regarded as an envelope function. In another words, the exciton wavefunctions can be viewed as product of envelope function and the function strongly localized on each lattice site $\mathbf{R}$. In this basis the Hamiltonian $\mathrm{H}_{0}$ is diagonal with matrix elements which can be divided into two parts [39]:

$$
H_{m n, m^{\prime} n^{\prime} ; \mathbf{K}, \beta, \beta^{\prime}}=\left\langle\Phi_{m n}(\mathbf{K}, \beta)\left|H_{0}\right| \Phi_{m n^{\prime}}\left(\mathbf{K}, \beta^{\prime}\right)\right\rangle=H_{\beta \beta^{\prime}}(\mathbf{K})+F_{\beta \beta^{\prime}}(\mathbf{K})
$$

Here $H_{\beta \beta^{\prime}}(\mathbf{K})$ contains energies of electron and hole in the bands $n$ and $m$, energy of the ground state of the crystal, and direct Coulomb interaction; whereas $F_{\beta \beta^{\prime}}(\mathbf{K})$ contains terms arising from electron-hole exchange interaction and corrections to direct Coulomb interaction for the finite size of the particles and for the symmetry of the crystal. Namely:

$$
\begin{aligned}
H_{\boldsymbol{\beta} \boldsymbol{\beta}^{\prime}}(\mathbf{K})=\delta_{\boldsymbol{\beta} \boldsymbol{\beta}} E_{0}^{1} & \\
& +e^{i \mathbf{K} \cdot(\boldsymbol{\beta}-\boldsymbol{\beta} \prime) / 2} N^{-1 / 2} \sum_{\mathbf{k}} e^{i \mathbf{k} \cdot\left(\boldsymbol{\beta}-\boldsymbol{\beta}^{\prime}\right)} \\
& \times\left[W_{n}\left(\mathbf{k}+\frac{1}{2} \mathbf{K}\right)-W_{m}\left(\mathbf{k}-\frac{1}{2} \mathbf{K}\right)\right]-\delta_{\boldsymbol{\beta} \boldsymbol{\beta}^{\prime}} e^{2} / \epsilon \beta
\end{aligned}
$$

Here $W_{n, m}$ are the energies of an electron in the conduction (valence) band and $E_{0}^{1}$ is the ground state energy. And the $F_{\beta \beta^{\prime}}(\mathbf{K})$ is: 


$$
\begin{aligned}
F_{\boldsymbol{\beta} \boldsymbol{\beta}^{\prime}}(\mathbf{K}) & =-\left[\left\langle n \boldsymbol{\beta} m \mathbf{0}|g| n \boldsymbol{\beta}^{\prime} m \mathbf{0}\right\rangle-\frac{\delta_{\boldsymbol{\beta} \boldsymbol{\beta}^{\prime}} e^{2}}{\epsilon \beta}\right]+2 \delta_{M}\left\langle n \boldsymbol{\beta} m \mathbf{0}|g| m \mathbf{0} n \beta^{\prime}\right\rangle \\
& +\sum_{\mathbf{R} \neq 0} e^{i \mathbf{K} \cdot \mathbf{R}}\left[2 \delta_{M}\left\langle n \boldsymbol{\beta} m \mathbf{R}|g| m \mathbf{0} n\left(\mathbf{R}+\boldsymbol{\beta}^{\prime}\right)\right\rangle\right. \\
& \left.-\left\langle n \boldsymbol{\beta} m \mathbf{R}|g| n\left(\mathbf{R}+\boldsymbol{\beta}^{\prime}\right) m \mathbf{0}\right\rangle\right]
\end{aligned}
$$

Here $\delta_{M}=1$ for the spin-singlet state (total spin momentum $S=0$ ), and $\delta_{M}=0$ for spin-triplet states (total spin momentum $S=1$ ). The symbols $n \mathbf{0}, n \boldsymbol{\beta}, n \mathbf{R}+\boldsymbol{\beta}$, etc. stand for one-electron states from band $n$ localized at the site of arbitrary chosen origin $\mathbf{O}$, at $\boldsymbol{\beta}$ from the origin, and at $\mathbf{R}+\boldsymbol{\beta}$ from the origin respectively. Thus notations like $|n \boldsymbol{\beta} m \mathbf{0}\rangle$ mean a hole at origin $\mathbf{O}$ in the band $m$ with an electron separated at $\boldsymbol{\beta}$ and belonging to band $n$, etc. $g$ has form of $g=$ $e^{2} / \epsilon\left|\mathbf{r}_{1}-\mathbf{r}_{2}\right|$ where $\mathbf{r}_{1}$ and $\mathbf{r}_{2}$ mean positions of the interacting particles. We note, that already without knowing exact form of the exciton wavefuntions, some conclusions can be made from form of $H_{\boldsymbol{\beta} \boldsymbol{\beta}^{\prime}}(\mathbf{K})$ and $F_{\boldsymbol{\beta} \boldsymbol{\beta}^{\prime}}(\mathbf{K})$. In the first approximation the exciton energy levels are obtained by addition the crystal's ground state energy, the energy build up from collective energy of electrons and holes and direct Coulomb interaction. Due to electron-hole exchange interaction the energies of spin-singlet and spin-triplet states are separated. The exchange term $\left\langle n \boldsymbol{\beta} m \mathbf{0}|g| m \mathbf{0} n \beta^{\prime}\right\rangle$ describes electron-hole exchange interaction when the hole and the electron are located in the same primitive cell and called short-range electron-hole exchange interaction, whereas the last term of $F_{\boldsymbol{\beta} \boldsymbol{\beta}^{\prime}}(\mathbf{K})$ describes electron-hole exchange interaction when the carriers in different primitive cells and called long-range electron-hole exchange interaction. We study in more details the electron-hole exchange interaction later in this section.

Now we are going to obtain eigenfunctions of $H_{0}$ by finding a unitary transformation which diagonalizes $H_{\boldsymbol{\beta} \boldsymbol{\beta}^{\prime}}(\mathbf{K})$ by substitution (3.4) into (3.8). Under assumption of the parabolic nondegenerate bands and assuming the coefficients $U$ in (3.4) are in the form of $U=e^{i \alpha \mathbf{K} \cdot \boldsymbol{\beta}} F(\beta)$ $\left(\alpha=\left(m_{e}^{*}-m_{h}^{*}\right) /\left(m_{e}^{*}+m_{h}^{*}\right)\right.$, and $F$ is some function), the equation for the coefficients $U$ in (3.4) becomes [39]:

$$
\left[-\frac{\hbar^{2}}{2 \mu} \nabla^{2}-\frac{e^{2}}{\epsilon \beta}\right] U=\left[E-E_{0}^{1}-E_{G}-\frac{\hbar^{2} K^{2}}{2\left(m_{e}^{*}+m_{h}^{*}\right)}\right] F
$$

Here $E_{G}$ is crystal's bandgap, $m_{e}^{*}, m_{h}^{*}$ are the electron and hole effective masses, $\mu$ is their reduced mass. This equation resembles the Schödinger equation of a hydrogen atom, thus the solutions are properly modified $(Z \rightarrow 1 / \epsilon)$ hydrogenic functions $F_{n l m}$ (We will denote set of quantum numbers $n l m$ as $v$ in following). Thus the $H_{\boldsymbol{\beta} \boldsymbol{\beta}^{\prime}}(\mathbf{K})$ is diagonal in the basis:

$$
\Psi_{v \mathbf{K}}=\sum_{\beta} e^{i \alpha \mathbf{K} \boldsymbol{\beta}} F_{v}(\boldsymbol{\beta}) \Phi(\mathbf{K}, \boldsymbol{\beta})
$$


where $\Phi(\mathbf{K}, \boldsymbol{\beta})$ are defined by (3.1). The energy eigenvalues of $H_{\boldsymbol{\beta} \boldsymbol{\beta}^{\prime}}(\mathbf{K})$ obtain form:

$$
E_{v \mathbf{K}}^{0}=E_{0}^{1}+E_{G}-\frac{\mu e^{4}}{2 \epsilon^{2} \hbar^{2} s^{2}}+\frac{\hbar^{2} K^{2}}{2\left(m_{e}^{*}+m_{h}^{*}\right)}
$$

where $s$ is an integer number. This result shows that in the first approximation the exciton energy consists of set of discreet energy levels condensing to $E_{0}^{1}+E_{G}$, or the bandgap energy of $E_{0}^{1}$ is assumed to be zero. For each energy level $s$ the exciton has kinetic energy associated with its total mass $M=m_{e}^{*}+m_{h}^{*}$ and wavevector $\mathbf{K}$. The full energy of the exciton is given by matrix elements (3.7). $H_{m n, m^{\prime} n^{\prime} ; \mathbf{K}, \beta, \beta}$ in representation (3.11) becomes:

$$
H_{m n, m^{\prime} n^{\prime} ; \mathbf{K}, \beta, \beta^{\prime}}=\sum_{\boldsymbol{\beta}, \boldsymbol{\beta}^{\prime}} U_{v \mathbf{K}}^{*}(\boldsymbol{\beta}) U_{v^{\prime} \mathbf{K}}\left(\boldsymbol{\beta}^{\prime}\right)\left[H_{\boldsymbol{\beta} \beta^{\prime}}(\mathbf{K})+F_{\boldsymbol{\beta} \boldsymbol{\beta}^{\prime}}(\mathbf{K})\right]
$$

The first term $H_{\boldsymbol{\beta} \boldsymbol{\beta}^{\prime}}(\mathbf{K})$ is diagonal in this representation and has form of $\delta_{v v} E_{v \mathbf{K}}^{0}$, while the second term is simply correction to it and can be approximated as

$$
\begin{aligned}
F_{\boldsymbol{\beta} \boldsymbol{\beta}^{\prime}}^{\prime}(\mathbf{K}) \approx-\left[\left\langle v \mathbf{0} m \mathbf{0}|g| v^{\prime} \mathbf{0} m \mathbf{0}\right\rangle-\sum_{\boldsymbol{\beta}} F_{v}^{*}(\boldsymbol{\beta}) F_{v^{\prime}}(\boldsymbol{\beta}) e^{2} / \epsilon \beta\right] \\
+2 \delta_{M} F_{v}^{*}(0) F_{v^{\prime}}(0)\langle n \mathbf{O} m \mathbf{0}|g| m \mathbf{0} n \mathbf{0}\rangle \\
+\sum_{\mathbf{R} \neq 0} e^{i \mathbf{K} \cdot \mathbf{R}}\left[2 \delta_{M}\left\langle v \mathbf{R} m \mathbf{0}|g| m \mathbf{R} v^{\prime} \mathbf{0}\right\rangle-\left\langle v \mathbf{R} m \mathbf{0}|g| v^{\prime} \mathbf{O} m \mathbf{R}\right\rangle\right]
\end{aligned}
$$

The first term in (3.14) in the rectangular brackets corrects the direct electron-hole Coulomb interactions for the finite area of localization of particles and for the crystal symmetry. The last two terms in (3.14) describe the electron-hole exchange interaction discussed in more details below.

The results above show that the very rough picture of a Wannier exciton can be obtained by ignoring all particles in the system except for a pair of excited electron and hole. The effect of the remaining particles is to create an effective potential in which the electron and the hole propagate giving them effective masses $m_{e}^{*}, m_{h}^{*}$. They interact according to the Coulomb law as modified with the medium with dielectric constant $\varepsilon$. Furthermore exciton motion can be decomposed into the center of mass motion with mass $M=m_{e}^{*}+m_{h}^{*}$ and wavevector $\mathbf{K}$, and the relative motion of the particles which resembles that of proton and electron in a hydrogen atom. The exciton wavefucntion is a product of envelope hydrogenic-like wavefunction and a function strongly localized a lattice sites $\mathbf{R}$ where the envelope (i.e. hydrogenic-like function) describes relative motion of the electron and the hole. Therefore, in many cases it is enough to consider only envelope wavefuntion and, consequently, consider the hydrogenlike model of an exciton constructed from carriers with the effective masses $m_{e}^{*}$ and $m_{h}^{*}$. This 
approximation is named the effective mass approximation and simplifies calculations drastically.

More rigorous treatment shows that the exciton wavefunction is in reality constructed from many single-particle wavefunctions. Therefore, the exciton is subjected to exchange interaction arising from indistinguishability of single particles. This interaction splits spin-singlet and spin-triplet states and can be in case of bulk excitons naturally divided into the shortand long- range parts.

Let consider the exchange interaction between two exciton states $\Psi_{\nu \mathbf{K}}=$ $\sum_{\beta} e^{i \alpha \mathbf{K} \boldsymbol{\beta}} F_{v}(\boldsymbol{\beta}) \Phi(\mathbf{K}, \boldsymbol{\beta})$ and $\widetilde{\Psi}_{v \mathbf{K}}$ constructed from sets of Wannier functions $\left\{a_{i}, b_{j}\right\}$ and $\left\{\tilde{a}_{i}, \tilde{b}_{j}\right\}$ respectively. Here $a_{i}\left(b_{j}\right)$ are Wannier functions of electron in a valence (conduction) band. Then the exchange interaction terms of (3.14) can be written in form [40]:

$$
J_{e x}=\delta_{\mathbf{K}, \mathbf{K}^{\prime}}\left(J_{D}+\iint \frac{d \mathbf{r}_{1} d \mathbf{r}_{2} \rho^{*}\left(\mathbf{r}_{1}\right) \tilde{\rho}\left(\mathbf{r}_{2}\right)}{\left\lfloor\mathbf{r}_{1}-\mathbf{r}_{2}\right\rfloor}\right)
$$

where: $J_{D}=\frac{4}{3}(\pi / \Omega)\left(3|\boldsymbol{\mu}||\mathbf{v}| \cos (\theta) \cos (\tilde{\theta})-\left(\boldsymbol{\mu}^{*}, \mathbf{v}\right)\right)$ with $\boldsymbol{\mu}=\int d \mathbf{r} \mathbf{r} \rho(\mathbf{r}) ; \boldsymbol{v}=\int d \mathbf{r} \mathbf{r} \tilde{\rho}(\mathbf{r})$ and $\rho(\mathbf{r})=\frac{-e}{\sqrt{N}} \sum_{\boldsymbol{\beta}} e^{i \alpha \mathbf{K} \boldsymbol{\beta}} F_{v}(\boldsymbol{\beta}) \sum_{i, j, \sigma} a_{i}(\mathbf{r}) b_{j}^{*}(\mathbf{r}+\boldsymbol{\beta})$. The first term in (3.15) is long-range part which is rewritten as dipole-dipole interaction and the second term is the short-range part. The short range splits the spin-singlet and spin-triplet components. In another words, effect of shortrange part is to split excitonic states of different symmetries. The long range part is related to dipole-dipole interaction. It corresponds to virtual creation and annihilation of the exciton on different atomic sites. This interaction causes splitting of the free exciton into longitudinal and transverse components. The former is the exciton with the dipole moment parallel to its wave vector $\mathbf{K}$. This part splits further spin-triplet states in ZB semiconductors [39], [41].

In cubic III-V materials the topmost valence band is 4 -fold degenerate and is constructed from $p$-like orbitals with the orbital angular momentum $L_{h}=1$. Therefore spin of electron in a valence band is not a good quantum number due to spin-orbit interaction. However the total angular momentum $J_{h}=L_{h}+s_{h}$, where $s_{h}$ is electron spin, remains good one. Thus the hole states (we exclude the split-off band) are $\left|J=\frac{3}{2} ; J_{z}= \pm \frac{3}{2}\right|_{h},\left|J=\frac{3}{2} ; J_{z}= \pm \frac{1}{2}\right|_{h}$, where $J_{h z}$ is the projection of the total angular momentum. However for s-like electrons of the conduction band spin quantum number is still good one. Therefore the spin-singlet exciton states in cubic III-V materials $\left|J ; J_{z}\right\rangle_{h} \otimes\left|s ; s_{z}\right\rangle_{e}$ are those with the exciton total angular momentum $J=J_{h z}+$ $s_{z e}=1$. The spin-triplet states are then those with the total angular momentum $J=2$. The former states can couple to radiative field, and thus their recombination is accompanied with the photon emission, and called bright excitons, while the latter states do not couple to the 
light field, and thus called dark excitons. Thus the exciton eigenstates in a $T_{\mathrm{d}}$ crystal are:

$J=1$ states:

$$
\begin{aligned}
& |1,1\rangle=\frac{\sqrt{3}}{2}\left|\frac{3}{2}, \frac{3}{2}\right\rangle|\downarrow\rangle-\frac{1}{2}\left|\frac{3}{2}, \frac{1}{2}\right\rangle|\uparrow\rangle \\
& |1,0\rangle=\frac{1}{\sqrt{2}}\left|\frac{3}{2}, \frac{1}{2}\right\rangle|\downarrow\rangle-\frac{1}{\sqrt{2}}\left|\frac{3}{2},-\frac{1}{2}\right\rangle|\uparrow\rangle \\
& |1,-1\rangle=\frac{1}{2}\left|\frac{3}{2},-\frac{1}{2}\right\rangle|\downarrow\rangle-\frac{\sqrt{3}}{2}\left|\frac{3}{2},-\frac{3}{2}\right\rangle|\uparrow\rangle
\end{aligned}
$$

$J=2$ states:

$$
\begin{aligned}
& |2,2\rangle=\left|\frac{3}{2}, \frac{3}{2}\right\rangle|\uparrow\rangle \\
& |2,1\rangle=\frac{\sqrt{3}}{2}\left|\frac{3}{2}, \frac{1}{2}\right\rangle|\uparrow\rangle+\frac{1}{2}\left|\frac{3}{2}, \frac{3}{2}\right\rangle|\downarrow\rangle \\
& \left.|2,0\rangle=\frac{1}{\sqrt{2}}\left|\frac{3}{2},-\frac{1}{2}\right| \uparrow\right\rangle+\frac{1}{\sqrt{2}}\left|\frac{3}{2}, \frac{1}{2}\right\rangle|\downarrow\rangle \\
& |2,-1\rangle=\frac{1}{2}\left|\frac{3}{2},-\frac{3}{2}\right\rangle|\uparrow\rangle+\frac{\sqrt{3}}{2}\left|\frac{3}{2},-\frac{1}{2}\right\rangle|\downarrow\rangle \\
& \left.|2,2\rangle=\left|\frac{3}{2},-\frac{3}{2}\right| \downarrow\right\rangle
\end{aligned}
$$

\subsection{Exciton bound to a defect center.}

\subsubsection{General considerations.}

The simplest bound exciton system consists of an electron and hole bound to ionized donor or acceptor. If we neglect effects of band degeneracy and effective mass anisotropy, the Hamiltonian for the exciton bound to a shallow donor becomes, if length is in units of $a^{*}=$ $\epsilon \hbar^{2} / m_{e}^{*} e^{2}$ and energy in units of $e^{2} / \epsilon_{0} a^{*}[41]$ :

$$
H=-\frac{1}{2} \xi \nabla_{h}^{2}-\frac{1}{2} \nabla_{e}^{2}+\frac{1}{r_{h}}-\frac{1}{r_{e}}-\frac{1}{\left|\mathbf{r}_{e}-\mathbf{r}_{h}\right|}=H_{0}+\xi H_{1}
$$

where: $\xi=m_{e}^{*} / m_{h}^{*}$. Then the total energy $\mathrm{E}$ is a monotonically increasing function of the mass ratio [41]:

$$
\frac{\partial E}{\partial \xi}=\left\langle\Psi(\xi)\left|H_{1}\right| \Psi(\xi)\right\rangle
$$

where $\Psi(\xi)$ is eigenfunction of $H$. Therefore for large $\xi$ the kinetic energy necessary to localize a hole at a donor becomes higher than the gain in energy upon the localization of the pair. Therefore there is some critical value for $\xi$ above which the complex is unstable. There are numerous attempts in the past to estimate the value of $\xi$. The efforts are characterized by 
different choices of the trial function and resulted in rather large range of $\xi$. The first calculations resulted in $\xi$ varied from 0.2 to 0.7 for excitons bound to ionized donors. More sophisticated calculations using methods for atomic calculations yielded in $\xi \approx 0.45$ [41].

In case of excitons bound to ionized acceptors or neutral donors theoretical calculations resulted in $\xi \approx 1$. It can be qualitatively explained as decrease of binding energy when $\mathrm{m}_{\mathrm{e}} \sim \mathrm{m}_{\mathrm{h}}$ due to increased electron-hole correlation energy, and free exciton state becomes the one with lower energy [41].

Isoelectronic impurities can bind an exciton. If the impurity atom has higher electronegativity that the atom it replaces, it will attract an electron. The long-range Coulomb potential of the trapped electron will attract a free hole, and the bound exciton will be formed. The simplest model, which does not account for lattice relaxation and detailed impurity potential, shows that the bound state exists when it satisfies condition [41]:

$$
1+\frac{J}{\bar{E}} \leq 0
$$

Here $J$ is the diagonal matrix element of the impurity potential in basis of the Wannier function of the band which dominates the impurity wave function, and $\bar{E}$ is the average energy of the band.

\subsubsection{Exciton bound to nitrogen-nitrogen pairs in GaP.}

Doping of bulk GaP by $\mathrm{N}$ induces formation of three types of bound excitons [42]. The simplest one is an exciton bound to a single $\mathrm{N}$ atom. Due to exchange interaction (often called $J$ - $J$ coupling), the exciton states are split into the $J=1$ triplet and the $J=2$ quintet states. The value of the splitting between these states is found to be equal to $0.91 \mathrm{meV}$ [43]. The transitions between the excitonic states and the crystal ground state with $J=0$ results in a strong A line (dipole allowed) and a weak, if observed, B-line (dipole forbidden). The second sort of the bound exciton is an excitonic molecule bound to the isolated nitrogen atom.

We will consider in this section the third sort of the bound excitons: an exciton bound to a $\mathrm{N}-\mathrm{N}_{i}$ pair. This is a single exciton bound to different pairs of $\mathrm{N}$ atoms. Index $i$ represents the separation between the $\mathrm{N}$ atoms, e.g. $i=1$ corresponds to two $\mathrm{N}$ atoms located at the closest possible $\mathrm{P}$ sites. The nitrogen potential in $\mathrm{GaP}$ is rather short-ranged and estimated to be confined within the lattice constant [43]. The local crystal field around a single $\mathrm{N}$ atom, which can be treated as infinite separation of atoms in a N-Ni pair, remains $\mathrm{T}_{\mathrm{d}}$. However in case of the $\mathrm{N}-\mathrm{N}_{i}$ pairs with finite separations the local symmetry of the crystal field is no longer $\mathrm{T}_{\mathrm{d}}$. The local symmetries of the N-Ni pairs were described by B. Gil et al in [44]-[46]. Electronic 
structure of the induced states will be determined by a combined effect of exchange interaction and HH- LH splitting driven by a local strain field.

As was discussed in the first chapter, in cubic crystals belonging to $\mathrm{T}_{\mathrm{d}}$ symmetry the topmost valence band at the center of BZ is 3-fold degenerate without inclusion of electron spin and $p$-like. The local field surrounding the bound exciton lifts the degeneracy of $\left|p_{x^{\prime}}\right\rangle,\left|p_{y^{\prime}}\right\rangle$, and $\left|p_{z^{\prime}}\right\rangle$ states (in the set of the local symmetry axis). In the simplest case of the uniaxial pair, the degeneracy of the $\left|p_{\alpha^{\prime}}\right\rangle, \alpha=x, y, z$, states is partly lifted by decoupling the $\left|p_{z^{\prime}}\right\rangle$ states from $\left|p_{x^{\prime}}\right\rangle,\left|p_{y^{\prime}}\right\rangle$. Including spin, one finds that the light hole states gain $\epsilon_{1}$ energy, and the heavy hole ones gain $\left(-\epsilon_{1}\right)$ energy. Here $\epsilon_{1}=\left\langle p_{x^{\prime}}|U| p_{x^{\prime}}\right\rangle=\left\langle p_{y^{\prime}}|U| p_{y^{\prime}}\right\rangle$, U is the local crystal field potential. Thus for positive values of the matrix element $\epsilon_{1}$ the LH states move up in energy by the value $\epsilon_{1}$ and the $\mathrm{HH}$ states lower their energy by value $-\epsilon_{1}$, therefore the total splitting of the HH-LH states is $2 \epsilon_{1}$ [44].

Inclusion of the exchange interaction results in the following exciton Hamiltonian matrix elements [43], [44]:

\begin{tabular}{c|ccccc} 
& $|2, \pm 2\rangle$ & $|2, \pm 1\rangle$ & $|2,0\rangle$ & $|1, \pm 1\rangle$ & $|1,0\rangle$ \\
\hline$\langle 2, \pm 2|$ & $-3 \gamma / 8+\epsilon_{1}$ & 0 & 0 & 0 & 0 \\
$\langle 2, \pm 1|$ & 0 & $-3 \gamma / 8-\epsilon_{1} / 2$ & 0 & $\pm \epsilon_{1} \sqrt{3} / 2$ & 0 \\
$\langle 2,0|$ & 0 & 0 & $-3 \gamma / 8-\epsilon_{1}$ & 0 & 0 \\
$\langle 1, \pm 1|$ & 0 & $\pm \epsilon_{1} \sqrt{3} / 2$ & 0 & $5 \gamma / 8+\epsilon_{1} / 2$ & 0 \\
$\langle 1,0|$ & 0 & 0 & 0 & 0 & $5 \gamma / 8+\epsilon_{1}$
\end{tabular}

Here $\gamma$ is the magnitude of the exchange interaction. It is evident that exciton states $|2,1\rangle$ and $|1,1\rangle,|2,-1\rangle$ and $|1,-1\rangle$ are mixed, and are not anymore the eigenstates of the unperturbed exciton Hamiltonian of $T_{d}$ symmetry. Total angular momentum $J$ is no longer a good quantum number, but $J_{z}$ is still good one. The resulting energy eigenvalues are:

$$
\begin{aligned}
& E_{A_{ \pm 1}}=\frac{\gamma}{8}+\frac{1}{2} \sqrt{\gamma^{2}+2 \gamma \epsilon_{1}+4 \epsilon_{1}} \\
& E_{B_{ \pm 1}}=\frac{\gamma}{8}-\frac{1}{2} \sqrt{\gamma^{2}+2 \gamma \epsilon_{1}+4 \epsilon_{1}}
\end{aligned}
$$

States $|1,0\rangle,|2,0\rangle,|2, \pm 2\rangle$ remain eigenstates with the energy eigenvalues:

$$
\begin{gathered}
E_{A_{0}}=\frac{5 \gamma}{8}-\epsilon_{1} \\
E_{B_{0}}=-\frac{3 \gamma}{8}-\epsilon_{1} \\
E_{B_{ \pm 2}}=-\frac{3 \gamma}{8}+\epsilon_{1}
\end{gathered}
$$


The results are plotted on Figure 7 the as a function of the $\epsilon_{1} / \gamma$.

In case of biaxial field degeneracy of exciton levels is further lifted and finally in case of general local filed of lower symmetry all exciton states are mixed providing with all possible transitions. These cases are discussed in details in [44], [47]. We can summarize the results in the Figure 8. An exciton bound to a single $\mathrm{N}$ atom neglecting the exchange interaction is shown on Figure 8a. The exchange interaction in $\mathrm{T}_{\mathrm{d}}$ symmetry splits the degenerate levels depicted on Figure 8a into 2 levels as seen on Figure 8b. Further decreasing of symmetry results in appearance more and more allowed transitions due to mixture of the dark states with bright states as seen on Figure $8 c, d$,e. The degree of admixture depends on the relative strength of local crystal field and the exchange interaction as well on the symmetry [44].

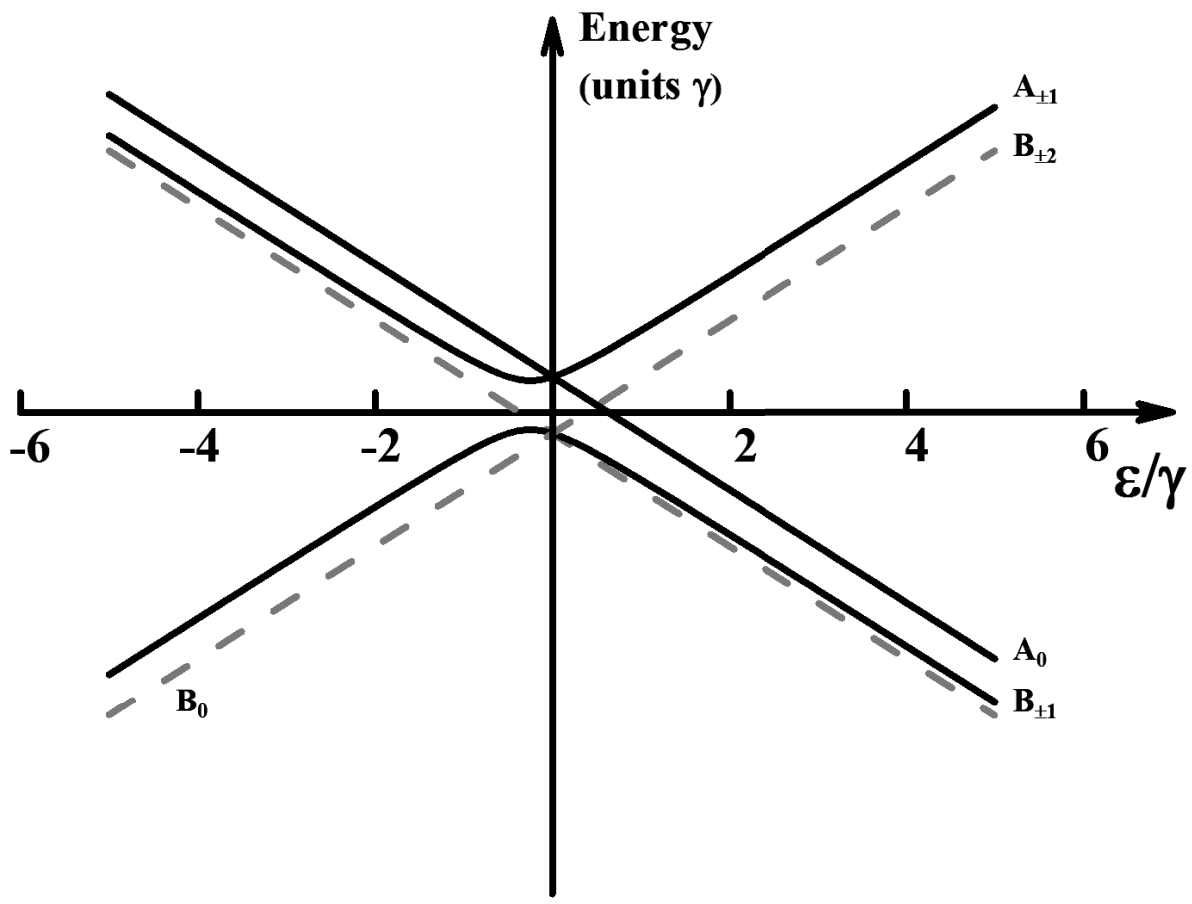

Figure 7. Splitting pattern of an exciton bound to a uniaxial center as a function of uniaxial potentialexchange energy ratio ( $\varepsilon$ and $\gamma$, respectively). The dashed lines represent forbidden transitions, the solid ones - dipole allowed transitions. Adopted from Ref. [44]. 


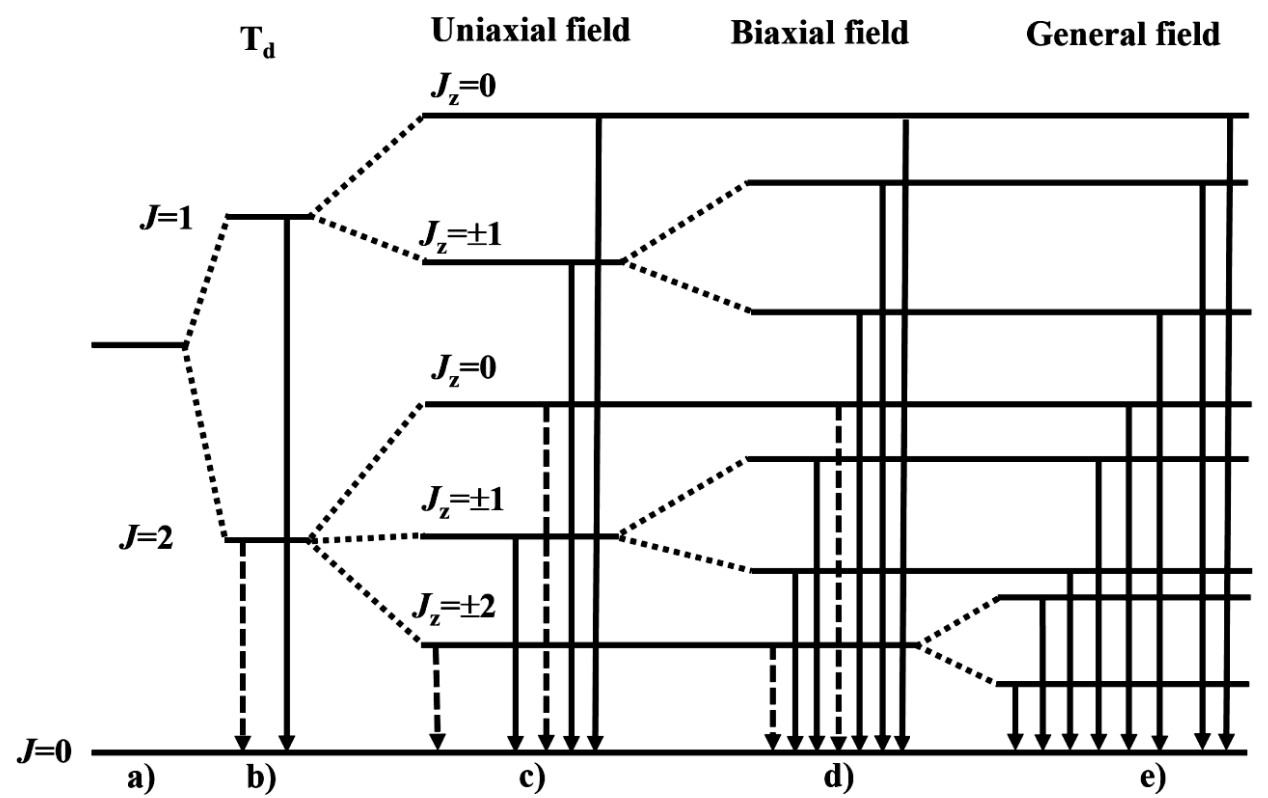

Figure 8. Schematic diagram of the J-J split exciton energy levels bound to a N-related center in case of compressive strain. a) an exciton bound to a single $N$ atom neglecting the J-J coupling. $b$ ) an exciton bound to a single $N$ atom accounting for the J-J coupling. $c$ ) exciton bound to a highly symmetrical N-related center with local field of $C_{3 v}$ or higher symmetry. d) excitonic energy levels in case of a $N$-center possessing a biaxial symmetry with local field of $C_{2 v}$ symmetry. e) excitonic energy levels when the exciton is bound to a N-related center of low symmetry. Solid (dashed) arrows represent dipole-allowed (dipole-forbidden) transitions. Adopted from Ref. [44].

\subsection{Exchange interaction in zero dimensional semiconductor structures.}

Exchange interaction in semiconductor quantum dots (QDs) and related to it exciton fine structure (FSS) splitting are field of controversy. In this section we will discuss first the long and short-range parts of the exchange interaction in QDs. Following references [48], [49] one can express the electron-hole exchange interaction as:

$$
H_{e h}=\sum_{\{i, j\}} C_{j_{2}, i_{1}, j_{3}, i_{4}}^{h e h e} a_{i_{1}}^{+} b_{j_{2}}^{+} b_{j_{3}} a_{i_{4}}
$$

where:

$$
C_{n_{1}, n_{2}, n_{3} n_{4}}^{p_{1}, p_{2}, p_{4}}=\iint d^{3} \mathbf{r}_{1} d^{3} \mathbf{r}_{2} \psi_{n_{1}}^{p_{1}{ }^{*}}\left(\mathbf{r}_{1}\right) \psi_{n_{2}}^{p_{2}{ }^{*}}\left(\mathbf{r}_{2}\right) \frac{e^{2}}{\epsilon\left|\mathbf{r}_{1}-\mathbf{r}_{2}\right|} \psi_{n_{3}}^{p_{3}}\left(\mathbf{r}_{2}\right) \psi_{n_{4}}^{p_{4}}\left(\mathbf{r}_{1}\right)
$$

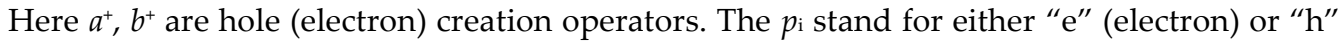


(hole), $n_{\mathrm{i}}$ runs over the corresponding single-particle wavefunctions.

In order to identify the matrix elements of the electron-hole exchange interaction Hamiltonian, one need to derive the appropriate wave functions. Numerous methods to perform that can be organized into two groups. The first group includes atomistic approaches, like pseudopotential methods [50]-[52] or tight-binding method [53], [54]. These methods consider the full atomic potential, several bands (light/heavy holes, split off band), etc. Thus they obtain the full wavefunctions, however those are purely numerical methods, computing costly and do not provide analytical expressions.

The second group is based on the effective mass approximation within the $\mathbf{k p}$ method framework [49], [55]-[58]. Thus the problem is reduced in this approach to finding the envelope functions as function of position. In general case one should include both $\mathrm{LH}$ and $\mathrm{HH}$ states. In relevant to this thesis case of InAs QDs embedded into GaAs significant compressive strain (experienced by the InAs QDs) will induce large splitting of $\mathrm{HH}$ states from LH states. Therefore in the consequent treatment we will include only $\mathrm{HH}$ states. In this approximation equation (3.23) can be written as:

$$
\begin{aligned}
& C_{n_{1}, n_{2}, n_{3}, p_{4}}^{p_{1}, p_{2}, p_{3}, p_{4}} \\
& s_{1}, s_{2}, s_{3}, s_{4} \\
& =\sum_{\mathbf{R}_{1}, \mathbf{R}_{2}} \phi_{n_{1}}^{p_{1} *}\left(\mathbf{R}_{1}\right) \phi_{n_{2}}^{p_{2} *}\left(\mathbf{R}_{2}\right) \phi_{n_{3}}^{p_{3}{ }^{*}}\left(\mathbf{R}_{2}\right) \phi_{n_{4}}^{p_{4} *}\left(\mathbf{R}_{1}\right) \\
& \times \iint \frac{e^{2}}{\epsilon\left|\mathbf{R}_{1}-\mathbf{R}_{2}+\mathbf{l}_{1}-\mathbf{l}_{2}\right|} u_{p_{1}, s_{1}}^{*}\left(\mathbf{l}_{1}\right) u_{p_{2}, s_{2}}^{*}\left(\mathbf{l}_{2}\right) u_{p_{3}, s_{3}}\left(\mathbf{l}_{2}\right) u_{p_{4}, s_{4}}\left(\mathbf{l}_{1}\right) d^{3} \mathbf{l}_{1} d^{3} \mathbf{l}_{2}
\end{aligned}
$$

In addition to set $\left\{p_{\mathrm{i}}, n_{\mathrm{i}}\right\}$ defined above, $s_{\mathrm{i}}$ denote spin; $\mathbf{r}=\mathbf{R}+\mathbf{l}, \mathbf{R}$ is the position of a primitive cell, 1 defines position inside the cell; $\phi_{n_{i}}^{p_{i}}$ and $u_{p_{i}, s_{i}}$ are envelope and Wannier-like singleparticle functions respectively.

We consider 2 cases: $\left|\mathbf{R}_{1}-\mathbf{R}_{2}\right|<\left|\mathbf{l}_{\mathbf{1}}-\mathbf{l}_{2}\right|$ (short-range subspace) and $\left|\mathbf{R}_{1}-\mathbf{R}_{2}\right|>\left|\mathbf{l}_{\mathbf{1}}-\mathbf{l}_{2}\right|$ long range subspace. The key part here is to approximate term $1 /\left|\mathbf{R}_{1}-\mathbf{R}_{2}+\mathbf{l}_{1}-\mathbf{l}_{2}\right|$ in (3.24) depending on regime (short- or long-range subspaces). The short-range exchange (SRE) part originates from approximation:

$$
\frac{1}{\left|\mathbf{R}_{1}-\mathbf{R}_{2}+\mathbf{l}_{1}-\mathbf{l}_{2}\right|} \approx \frac{1}{\left|\mathbf{l}_{1}-\mathbf{l}_{2}\right|} v_{o} \delta\left(\mathbf{R}_{1}-\mathbf{R}_{2}\right)
$$

For the long-range exchange (LRE) part the $1 /\left|\mathbf{R}_{1}-\mathbf{R}_{2}+\mathbf{l}_{1}-\mathbf{l}_{2}\right|$ can be expanded in multipole series giving rise to LRE: 


$$
\begin{aligned}
\frac{1}{\mid \mathbf{R}_{1}-\mathbf{R}_{2}+\mathbf{l}_{1}-} & \mathbf{l}_{2} \mid \\
& \approx \frac{1}{\left|\mathbf{R}_{1}-\mathbf{R}_{2}\right|}-\frac{\mathbf{R}_{1}-\mathbf{R}_{2}}{\left|\mathbf{R}_{1}-\mathbf{R}_{2}\right|^{3}}\left(\mathbf{l}_{1}-\mathbf{l}_{2}\right) \\
& +\frac{1}{2}\left(\mathbf{l}_{1}-\mathbf{l}_{2}\right) \frac{3\left(\mathbf{R}_{1}-\mathbf{R}_{2}\right)^{2}-\mathbf{I}}{\left|\mathbf{R}_{1}-\mathbf{R}_{2}\right|^{5}}\left(\mathbf{l}_{1}-\mathbf{l}_{2}\right)+\cdots
\end{aligned}
$$

Here I stands for the identity matrix. The first two terms describe monopole-monopole and monopole-dipole interactions which give vanishing exchange interaction due to orthogonality of the single-electron functions and exclusion of band mixing. The first non-vanishing term is the dipole-dipole interaction. The elements of $C_{n_{1}, n_{2}, n_{3}, n_{4}}^{h e h}$ of (3.24) matrix can be arranged as:

\begin{tabular}{c|cccc}
$\left|\frac{3}{2},-\frac{1}{2}\right|$ & $\left|\frac{3}{2},-\frac{1}{2}\right\rangle$ & $\left|-\frac{1}{2}, \frac{3}{2}\right\rangle$ & $\left.\frac{1}{2}, \frac{3}{2}\right\rangle$ & $\left|-\frac{3}{2},-\frac{1}{2}\right\rangle$ \\
\hline $\begin{array}{c}\left\langle-\frac{3}{2}, \frac{1}{2}\right. \\
\Delta_{0}\end{array}$ & $\frac{1}{2} \Delta_{1}^{*}$ & $\Delta_{0}$ & 0 & 0 \\
$\left\langle\frac{3}{2}, \frac{1}{2}\right|$ & 0 & 0 & 0 & 0 \\
$\left\langle-\frac{3}{2},-\frac{1}{2}\right|$ & 0 & 0 & $\frac{1}{2} \Delta_{2}^{*}$ & $\frac{1}{2} \Delta_{2}$
\end{tabular}

Terms of form $\Delta_{0}$ are often called the "isotropic electron-hole exchange interaction". These elements are not sensitive to the in plane asymmetry of the confinement potential and split spin-singlet states from spin-triplet states. (In cubic III-V materials these are $J=1$ and $J=2$ exciton states) These terms contain SRE and the "out-of-plane" component of LRE. Contrary, in bulk only SRE splits states of different symmetry. The detailed expression of $\Delta_{0}$ is given in [48]. The bright states are degenerate, and their recombination results in emission of the same energy, but of the different chirality.

Terms $\Delta_{2}$ split the states with parallel pseudospins. In the cubic III-V materials this part of the exchange interaction splits the dark states. These terms vanish in the one electron approximation.

Terms $\Delta_{1}$ are called "anisotropic" electron-hole interaction. In the single band approximation this part is purely long-range one. LRE mixes the exciton states with antiparallel pseudospin states. The resulting state becomes $(|\Uparrow \downarrow\rangle \pm|\Downarrow \uparrow\rangle) / \sqrt{2}$, (where $\Uparrow$ denotes hole pseudospin, and $\uparrow$ denotes electron spin), and the degeneracy of these states is lifted. In cubic III-V materials it means that the bright states are split. The recombination of the split states gives 
rise to two linearly polarized components.

Origin of LRE in QDs was first studied by Takagahara [55]. Within the single band approximation it was showed that the long-range part of the exchange interaction disappears in spherically symmetrical dots. More elaborated calculations showed that any reduction from a laterally isotropic confinement potential (i.e. deviation from $C_{\infty \mathrm{v}}$ ) results in appearance of the LRE, which causes mixing and splitting of the $J=1$ exciton states. In another words the origin of the LRE was ascribed to asymmetry of the confinement potential. It was also found that the lower energy exciton component is polarized along the elongation axis of the confinement potential [56]-[58]. However the nature of the LRE part (i.e. dipole-dipole interaction) was not questioned in these works. Many-body atomistic pseudopotential calculations showed the LRE has significantly different nature in the QDs than in bulk [50]. The LRE is dominated by the monopole-monopole interaction due to non-orthogonality of the single particle functions. Thus LRE can't be ascribed to simple dipole-dipole interaction between different primitive cells. This rises question if SRE already mixes the bright states given the confinement potential is asymmetric enough, and if so, what is balance between SRE and LRE.

This relative importance of the LRE and SRE in mixing of bright states is an open question. In works [54]-[58] the LRE was shown to be responsible for the for the bright exciton doublet structure. However several works consider only SRE [59], [60]. Atomistic calculations showed that the balance between SRE and LRE changes as function of the dot shape and the type (selfassembled, thickness fluctuation, etc.) [52].

Thus LR and SR parts of electron-hole exchange interaction and their contribution to the splitting of the bright states are still under discussion. However it is generally accepted that the lowering symmetry of the confinement potential causes the splitting. Group theory analysis shows that if symmetry of the confinement potential is lower than $\mathrm{C}_{3 \mathrm{v}}$ the bright exciton states belong to 2 different irreducible representations, and thus split in energy [61], [62]. Lowering of symmetry of the confinement potential can be induced by several factors. In selfassembled QDs one can identify several major contributors.

One of the most important factors inducing lowering of the confinement potential is strain induced piezoelectricity (PZ) [61], [63]-[67]. This factor is widely believed to be responsible for rather large observed splittings of tens and hundreds of $\mu \mathrm{eV}$. The self-assembled InAs dots are typically grown on (001) GaAS substrate. Ga-As "dimers" are arranged in rows along [110], leaving "free" trenches in between [68]. The In atoms upon deposition will therefore tend to diffuse more in [110] thus causing elongation of the QDs along the direction. This results in appearance of stress along [110] due to lattice mismatch between GaAs and InAs 
and appearance of the PZ field. When only first order PZ is included, the PZ filed is predicted to be along [110], i.e. elongation of the QD [66], [67]. This preferential direction of PZ will result in break of the rotational symmetry of the confiment potential. However it was shown by pseudopotential calculations that the second-order PZ terms are not neglectable in the QDs and their magnitude can be as large as the first order terms. In the perfect lens-shaped dots they almost cancel each other [65]. Later calculations showed that the balance between the first and second order terms depends on the QDs actual shape [64].

The degree of geometrical asymmetry is also widely considered as responsible for rather large splitting [64], [67], [69]-[72]. Obviously spatially elongated QD will also have asymmetrical condiment potential. However in the self-assembled QDs as we already have seen, the asymmetry and PZ are linked to each other. In case of directional PZ field the electrons and holes will elongate along two orthogonal direction. In case of only first order PZ those are [110] and [11̄0] respectively [66]. However even the perfectly shaped dots are not necessary will have fully symmetric confinement potential. The set of so called intrinsic effects (atomistic symmetry, randomness of composition profile) will induce small perturbations of the confinement potential. These effects are predicted to contribute to the splitting lower than $10 \mu \mathrm{eV}$ [63], [64], [73], [74].

Thus though various factors causing the FSS are known, their relative importance is still under debate. Nevertheless PZ and shape asymmetry are widely believed to be the most important factors. In paper 5 we study effects of compensation of strain induced PZ and shape asymmetry. 


\section{Chapter 4 Polarization properties of semiconductor nan- owires.}

\subsection{Optical antenna effect.}

Due to a high mismatch of dielectric constants of the III-V NW material and the surrounding media and the high aspect ratio, the NWs often show strong polarization anisotropy in absorption and emission of radiation. Polarized absorption and emission were first observed in individual InP NWs in photoluminescence (PL) and photoconductivity measurements [75]. Polarization of absorption is typically seen as a strong dependence of the PL and photocurrent intensities on the polarization of the excitation light. Polarization anisotropy of the emission is observed as dependence of the emission intensity on an angular position of an analyzer [76], [77].

One can identify the following mechanisms responsible for this behavior [78]:

1. Modification of the energy spectrum and optical matrix elements due to spatial confinement of the carriers. The size quantization and the dielectric mismatch with the surrounding medium lead to modification of exciton parameters.

2. Dielectric confinement of the optical electric field due to mismatch in dielectric constants of the NW material and the outer medium.

The first class of effects is important in the strong confinement regime, where the NW radius is comparable with an exciton Bohr radius ( $10 \mathrm{~nm}$ in GaAs). This is the case only for the very thin NWs with diameters smaller than $20 \mathrm{~nm}$. In most cases, however, NWs have substantially larger diameters of around $60-300 \mathrm{~nm}$. Therefore, their polarization properties are driven by the second, purely classical, mechanism. The polarization response of the NWs is then calculated based on Maxwell equations using an image forces approach [78].

Ruda [78] modelled the NW emission assuming isotropic emitting dipoles $\mathbf{d}_{0}=$ $\left(d_{0 x}, d_{0 y}, d_{0 z}\right)$ located at the axis of the NW. The dielectric confinement modifies the dipole moments as:

$$
\begin{aligned}
& d_{x}=d_{0 x} \sqrt{\epsilon} \frac{J_{1}^{\prime}(k a) H_{1}^{(1)}(k a)-J_{1}(k a) H_{1}^{(1)^{\prime}}(k a)}{\sqrt{\epsilon} J_{1}^{\prime}(k a) H_{1}^{(1)}\left(k_{0} a\right)-\sqrt{\epsilon} J_{1}(k a) H_{1}^{(1)^{\prime}}\left(k_{0} a\right)} \\
& d_{z}=d_{0 z} \sqrt{\epsilon} \frac{J_{1}(k a) H_{0}^{(1)}(k a)-J_{0}(k a) H_{1}^{(1)}(k a)}{\sqrt{\epsilon} J_{1}(k a) H_{0}^{(1)}\left(k_{0} a\right)-\sqrt{\epsilon} J_{0}(k a) H_{1}^{(1)}(k a)}
\end{aligned}
$$

Here $a$ denotes the NW radius, $k=\sqrt{\epsilon} \omega / c, k_{0}=\sqrt{\epsilon_{0}} \omega / c$ are the wave vectors in the material 
(dielectric constant $\epsilon$ ) and in the air (dielectric constant $\epsilon_{0}$ ) respectively, $J_{m}\left(H_{m}^{(1)}\right)$ are the Bessel (Hankel) functions of the first kind, the prime stands for the derivative. The polarization of the emission is given then by expression:

$$
\frac{I_{\|}}{I_{\perp}}=\frac{d_{x}^{2}+2 d_{z}^{2}}{3 d_{x}^{2}}
$$

The results of these calculations for GaP NWs of various diameters are shown in Figure 9.

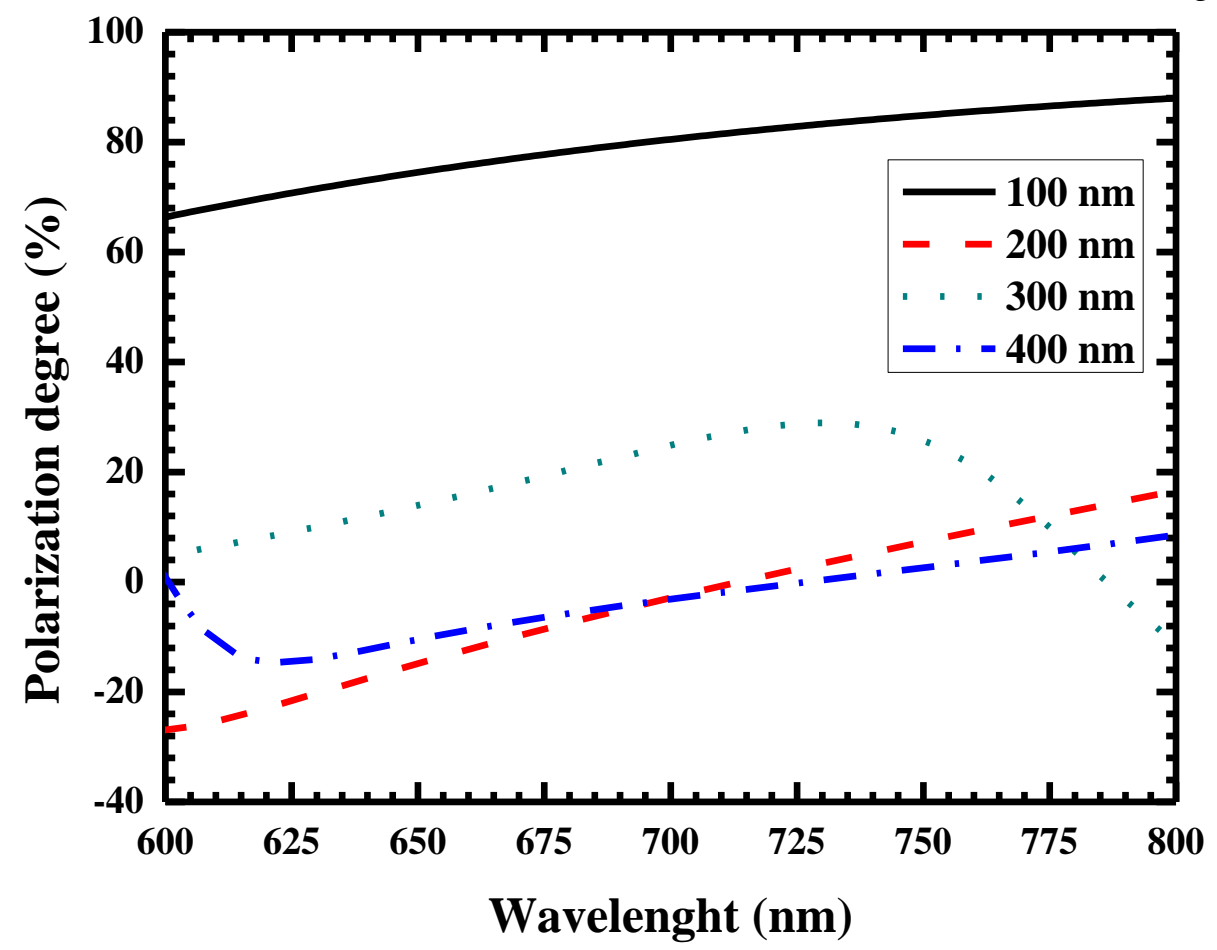

Figure 9. Calculated polarization degree according optical antenna effect for GaP NWs of various diameters. 


\subsection{Polarization properties of ZB and WZ nanowires.}

\subsubsection{Selection rules.}

Neglecting non-linear effects, the electromagnetic interaction couples an initial state $|i\rangle$ belonging to $\mathrm{VB}$ with the final state $|f\rangle$ belonging to $\mathrm{CB}$ via the Hamiltonian:

$$
H_{e m}^{\prime}=-\frac{e}{m c} \mathbf{p A}
$$

The transition occurs when the matrix element $\left\langle f\left|H_{e m}^{\prime}\right| i\right\rangle$ is nonzero. The matrix elements are scalar and, thus, under the crystal space group transformations transform as the fully symmetric representation $\Gamma_{1}$. In Eq. (4.3) $\mathbf{p}$ is the electron momentum and $\mathbf{A}$ is the vector potential of an external electromagnetic field. The momentum operator is a part of the system under consideration, however A acts like "reservoir" in thermodynamical sense. Thus p acts in the Schrödinger equation as an operator, and $\mathbf{A}$ does not. This means that $H_{e m}^{\prime}$ transforms as a vector. In case of cubic crystals, all the components $\left(p_{x}, p_{y}, p_{z}\right)$ of the vector transform as the same irreducible representation. However in crystals of lower symmetry the components transform according to different irreducible representations. For example, in $\mathrm{C}_{6 \mathrm{v}}$ group $\mathrm{p}_{\mathrm{x}}$ and $p_{y}$ transform as two-dimensional irreducible representation, whereas $p_{z}$ as the one-dimensional representation. The transition is possible if $\Gamma^{f} \subset \Gamma^{H_{e m}^{\prime}} \otimes \Gamma^{i}$, where $\Gamma^{i(f)}$ is the irreducible representation of initial/final states and $\Gamma^{H_{e m}^{\prime}}$ is the irreducible representation of $H_{e m}^{\prime}$. In case when polarization of the radiation is of interest, one needs to consider irreducible representation of the particular component of $\mathbf{p}$ which reflects the polarization of the radiation (say, along $x$ or $y$ direction) [6].

These group-theory considerations allow one to define allowed optical transitions in various semiconductors. For ZB structures these include the $\Gamma_{8 \mathrm{v}}-\Gamma_{6 \mathrm{c}}$ and $\Gamma_{7 \mathrm{v}}-\Gamma_{6 \mathrm{c}}$ transitions that are not polarized [7]-[9]. In WZ structures, VB splits two $\Gamma_{7 \mathrm{v}}$ and $\Gamma_{9 \mathrm{v}}$ subbands. The lowest in energy $\Gamma_{9_{v}}-\Gamma_{7 c}$ transition is allowed in the $E \perp c$ polarization, whereas the $\Gamma_{7 \mathrm{v}}-\Gamma_{7 \mathrm{c}}$ transitions do not show preferential polarization [8], [9], [79]. As a result, the emission of III-V WZ materials (GaAs, InP, etc.) is orthogonally polarized [79].

\subsubsection{Experimental observations.}

Impacts of crystalline structure on polarization of light emission were first studied by Mishra et al [80] for InP NWs. Light emitted from ZB NW was found to be polarized along the NW axis, as expected from the antenna effect. On the other hand, WZ NWs demonstrated the orthogonal polarization with respect of the NW axis, in accordance with the selection rules discussed above. However the degree of polarization was not $100 \%$ since the optical 
antenna effect in the thin NWs favors the parallel polarization restricting attainable polarization degrees. These results were afterwards confirmed for other material systems [81]-[83].

The ZB and WZ parts may coexist on a single NWs leading to the formation of polytypic heterojuntions. In GaAs and InP, the corresponding hetero-interfaces have type-II band alignment [84], [85] with holes (electrons) being confined within the WZ (ZB) regions. Therefore the band-to-band transitions at these heterointerfaces should exhibit the orthogonal polarization, as was indeed observed for InP and GaAs NWs containing alternating WZ and ZB inclusions [86]. In GaAs, the WZ and ZB polytypes are commonly agreed to have similar bandgap energies with the majority of recent papers favoring $E_{g}^{W Z}>E_{g}^{Z B}$ [87]-[89]. However the exact value of the band offset remains under discussion with the reported values varying from 35 to $100 \mathrm{meV}$ [87], [89], [90]. In such spontaneously formed polytypic heterostructures thicknesses of WZ and ZB segments can vary from several to hundreds of nanometers, which affects energies of the related type-II optical transitions. The latter form a rather broad emission band detected below the GaAs bandgap. 


\section{Chapter 5 Material systems.}

\subsection{ZnO.}

An excellent and comprehensive description of basic properties of $\mathrm{ZnO}$ is given in Ref. [91].

The thermodynamically stable crystal phase of $\mathrm{ZnO}$ under ambient condition is WZ. Therefore, $\mathrm{ZnO}$ lattice belongs to the $C_{6 v}^{4}$ space group with an ideal ratio between lattice parameters $c / a=\sqrt{8 / 3}$. The structure is composed of two interpenetrating hexagonal closed packed sublattices (hcp). Each of these sublatticese consists of atoms of the same kind and they are displaced in the direction of the $c$-axis by $u=3 / 8 c$ in case of ideal structure. Each sublattice includes 4 atoms per primitive cell. The real $\mathrm{ZnO}$ structure deviates from the ideal parameters due to differences in electro-negativities of the constituent atoms. The lattice parameters slightly vary between different reports, however the discrepancies are not very high, $a \approx$ $3.3 \AA ̊, c \approx 5.2 \AA$. Since $\mathrm{ZnO}$ is a binary tetrahedral semiconductor, each anion is surrounded by 4 cations and vice versa. This coordination is typical for $s p^{3}$ hybridized covalent bonding. However $\mathrm{ZnO}$ shows significant ionicity.

$\mathrm{ZnO}$ is a direct bandgap semiconductors with the bandgap energy of $3.43 \mathrm{eV}$ at RT. The valence band structure of $\mathrm{WZ}$ semiconductors was discussed earlier in this thesis. $\mathrm{In} \mathrm{ZnO}$, however, ordering of the $\mathrm{A}, \mathrm{B}, \mathrm{C}$ VB subbands $\left(\Gamma_{7}-\Gamma_{9}-\Gamma_{7}\right)$ is inverted as compared with that in the majority of WZ semiconductors, as was proved only most recently [92]-[94] following decades of extensive debates. This inverted ordering of the VB states is due to negative spinorbit interaction caused by energy proximity of $d$-orbitals of $\mathrm{Zn}$ [92]. The energy spacing between $\mathrm{A}$ and $\mathrm{B}$ (B and $\mathrm{C})$ bands is $\triangle \mathrm{E}_{\mathrm{AB}}=4.9 \mathrm{meV}\left(\triangle \mathrm{E}_{B \mathrm{C}}=43.7 \mathrm{meV}\right)$.

The free exciton binding energy in $\mathrm{ZnO}$ is $60 \mathrm{meV}$, which makes excitons stable even at room temperature. Group theory analysis shows that A-, B-, and C-excitons are dipole active, where A $(B, C)$ stands for the hole symmetry. Since CB in ZnO has $\Gamma_{7 \mathrm{c}}$ symmetry, the symmetries of excitons can be obtained as direct products of the corresponding irreducible representations:

$$
\begin{gathered}
\Gamma_{7} \otimes \Gamma_{9} \rightarrow \Gamma_{5} \oplus \Gamma_{6} \text { (A-exciton) } \\
\Gamma_{7} \otimes \Gamma_{7} \rightarrow \Gamma_{5} \oplus \Gamma_{1} \oplus \Gamma_{2} \text { (B,C-exciton) }
\end{gathered}
$$

In the absence of external perturbations the optical processes are governed by the Hamiltonian $H_{e m}^{\prime}$ introduced in section 4.2.1., which transforms as a vector. In double group of $\mathrm{C}_{6 \mathrm{v}}$ only $\Gamma_{5}$ and $\Gamma_{1}$ transform as vectors. Therefore all three excitons are allowed in polarization orthogonal to the $c$-axis, while A-exciton is symmetry forbidden in polarization parallel to the $c$-axis. 
Due to the presence of four atoms within a primitive cell, there are 12 phonon modes, 3 of them are acoustic (1 LA and 2 TA), and the rest are optical modes. Symmetries of the phonon modes at the $\mathrm{BZ}$ center are $\Gamma_{v i b}=2 A_{1} \oplus 2 E_{1} \oplus 2 B_{1} \oplus 2 E_{2}$. The acoustic modes transform as $A_{1}$ and $E_{1}$, thus the optical modes are $\Gamma_{v i b}^{o p t}=A_{1} \oplus E_{1} \oplus 2 B_{1} \oplus 2 E_{2}$. Modes of $A_{1}$ and $E_{1}$ symmetries are both IR and Raman active, while the $E_{2}$ modes are only Raman active and $B_{1}$ are silent. Due to anisotropy of the macroscopic electric field, the modes propagating along the $c$-axis $\left(A_{1}\right)$ and in plane $\left(E_{1}\right)$ are split in energy. They are additionally split into TO-LO modes. The modes of E2 symmetry at the center of the BZ represent primarily oscillations of oxygen atoms $\left(E_{2}^{H}\right)$ and of zin atoms $\left(E_{2}^{L}\right)$.

\subsection{InAs.}

InAs is a III-V compound semiconductor. This semiconductor and related alloys gained recent attention as active materials for a wide variety of electronic applications as well as IR sensitive photodetectors. In this section we follow mostly books of S. Adachi [95], [96], which contain comprehensive details of the material properties.

InAs crystallizes into ZB crystal structure belonging to $\mathrm{T}_{d}^{2}$ space group. The structure is composed of two interpenetrating fcc sublattices displaced along the body diagonal by one fourth of its length. Each sublattice consists of atoms of the same kind. Unlike the case of WZ structure, the lattice can be characterized by a single lattice constant of $6.06 \AA$. InAs like ZnO is a tetrahedral semiconductor with $s p^{3}$ hybridized covalent bonding that has a noticeable ionicity. InAs is a direct bandgap semiconductor with the room temperature bandgap energy of $0.359 \mathrm{eV}$. The valence band consists of three twofold degenerate subbands of light-, heavy, and split-off bands. At $\Gamma$ point the states of light and heavy holes are degenerate and transform as $\Gamma_{8}$. The split-off band is of $\Gamma_{7}$ symmetry and lays deeper in energy by $0.367 \mathrm{eV}$. The conduction band is of $\Gamma_{6}$ symmetry.

Due to cubic symmetry and the presence of two atoms in a primitive cell, the phonon modes have $\mathrm{T}_{2}$ symmetry at the $\Gamma$ point. The optical modes are split into $\mathrm{TO}$ and LO branches due to ionicity of the lattice. These modes are both IR and Raman active.

\section{3. $\mathrm{Ga}_{1-x} \mathrm{~N}_{x} \mathrm{P}$ and $\mathrm{Ga}_{1-\mathrm{x}} \mathrm{N}_{x} \mathrm{As}$ alloys.}

GaNP and GaNAs belong to the so-called dilute-nitride semiconductors. Incorporation of $\mathrm{N}$ in GaP and GaAs leads to a number of unusual effects summarized in [97]. One of the most prominent effects is the huge bandgap bowing and splitting of CB into two subbands: $\mathrm{E}$ - and 
$\mathrm{E}_{+}$[98], [99]. Another remarkable effect of $\mathrm{N}$ incorporation is a direct-indirect transition induced by nitrogen incorporation in GaP [99]-[102].

A simple and rather straightforward explanation of the huge bandgap bowing is provided by the band anticrossing (BAC) model [108]. The model assumes that the changes in the electronic structure are caused by an interaction between delocalized CB states of the host semiconductor and localized states of the isoelectronic impurity. In case of a highly electronegative impurity, such as $\mathrm{N}$ in III-V, localization of electrons occurs at states with $\mathrm{A}_{1}$ symmetry. Since the symmetry of the $C B$ is also $A_{1}$, the restructuring of the $C B$ of the host material happens. The quantative treatment is carried out by considering a two-band anticrossing within the many-impurity Anderson model. The dependency of the split CB edges are given by [98]:

$$
E_{ \pm}=\frac{1}{2}\left[\left(E_{g}+E_{N}\right) \pm \sqrt{\left(E_{g}-E_{N}\right)^{2}+4 V^{2} x}\right]
$$

Here $E_{g}$ is the bandgap of the original material, $E_{N}$ is the energy of localized states, $\mathrm{V}$ is the averaged hybridization strength between the $\mathrm{CB}$ and $\mathrm{N}$ states and is the fitting parameter of the theory, $x$ is $\mathrm{N}$ concentration.

This rather simple and straightforward model can fit remarkably well many parameters of dilute nitrides including temperature-, composition- and pressure-dependencies of their bandgap energy. The BAC model also predicts the nature of the CB minimum (CBM) states. It is known that in the ultra-dilute regime (the doping regime), the $\mathrm{N}$ atoms produce highly localized states [97], [103]. In GaAs, these states are resonant with CB states and located 180 meV above the CBM [97], [103]. Therefore, the BAC model considers that CB edge in GaNAs is formed from the CB states.

Later tight-binding (TB) calculations extended the BAC model by including also $\mathrm{N}$ cluster states [97], [104], [105]. It was found that with increasing [N] the CB edge sweeps the N-cluster states one by one and hybridize with them. Thus though CBM in GaNAs is formed mainly from the $\mathrm{CB}$ states, it has admixture of the $\mathrm{N}$ states resulting in a variation of the $\Gamma$-character with $[\mathrm{N}]$. The E+ states, on the contrary, are formed from N-related states.

The situation in GaNP is more complicated. The host GaP semiconductor has an indirect bandgap, and the isolated $\mathrm{N}$ atoms produce an electronic level located below the CBM [97], [103]. Thus BAC predicts that CBM states in GaNP evolve from the $\mathrm{N}$ localized states $E_{N}$. Experimentally, however, it was shown that the $E_{N}$ level does not change with $\mathrm{N}$ composition [97], [100], [106]. More consistent explanation for the bands formation was given from the first principle calculations [113]. It was concluded that the lowest $E$ - band is constructed due the 
$\mathrm{N}$-induced perturbation and mixing of the host states. The TB calculations [107] also suggested that hybridization with deep $\mathrm{N}$ cluster states may further contribute to the CBM formation. The formation of the higher $\mathrm{E}_{+}$band was explained as hybridization between higher lying CB states.

We summarize our discussion on schematic drawing presented in Figure 10.

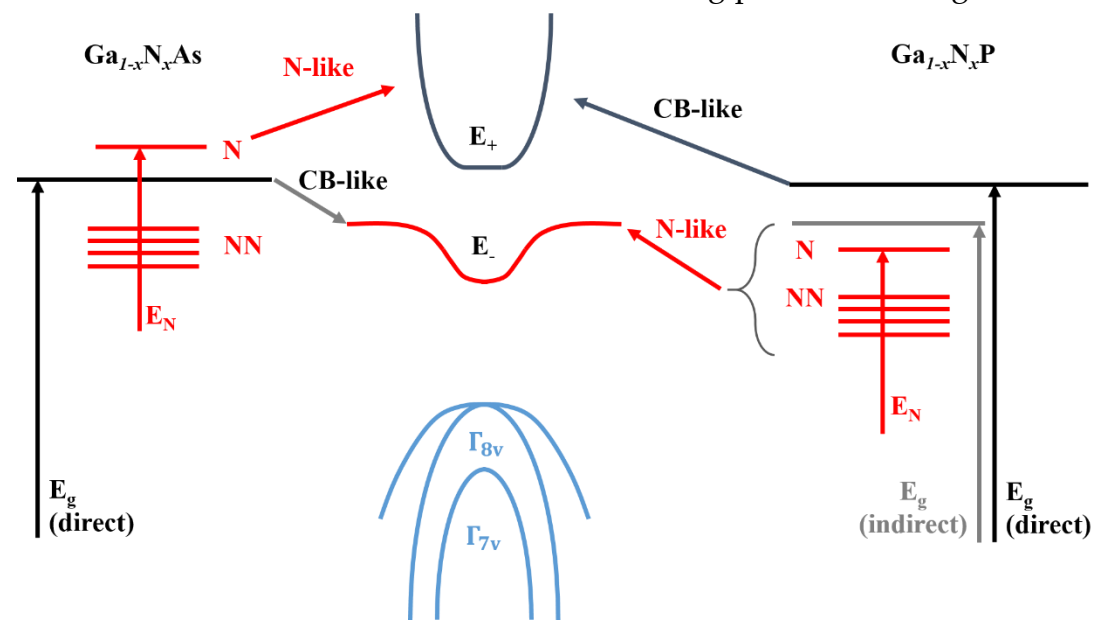

Figure 10. Schema of electronic structure of GaNAs and GaNP. N states for isolated N level while NN stands for various $N$-clusters. $N$-induced formation of $E_{-1+}$ states in the alloys is shown in the middle. The arrows link these bands with states they originate from according to the discussion above. 


\section{Chapter 6 Experimental setup and techniques.}

\subsection{Micro-photoluminescence ( $\mu-P L)$ spectroscopy.}

Photoluminescence (PL) spectroscopy is a widely used method to perform optical characterization of solids. In this method laser light is used to excite electrons from occupied to empty states via absorption of the incident photon. These photo-excited carriers can recombine with emission of a photon (radiative recombination) giving rise to the PL signal. Thus PL spectroscopy allows one to gain some information about electronic structure of the material.

In conventional PL spectroscopy, the excitation and detection of the PL signal employs ordinary lenses. While it is adequate for characterization of bulk materials, spatial resolution in such measurements is insufficient to study individual nanostructures as PL is excited and collected from a large number of individual structures. The situation is improved in microphotoluminescence ( $\mu$-PL) measurements where a microscope objective is used for the PL excitation and detection. This leads to submicron resolution, sufficient to optically distinguish optical responses from individual NWs or to perform single QD spectroscopy.

In this thesis, the $\mu$-PL measurements were performed using a confocal Horiba Jobyn-Yvon LabRAM 800 system schematically shown in Figure 11. This instrument consists of a coupled microscope, single grating monochromator and a set of two detectors (Peltier-cooled Si CCD and liquid nitrogen cooled InGaAs diode array). The excitation sources are solid-state lasers (a tunable Ti:sapphire laser or laser diodes) or a He-Cd laser. In order to control the excitation power, a set of rotary neutral density (ND) filters was used. The laser beam was collimated using two iris diaphragms. The PL excitation and detection were performed with the same objective lens in the backscattering geometry. Polarization of the detected PL signal was measured using polarization optics consisting of a rotary half wave plate and a fixed linear Glan-Thompson polarizer, which were inserted in the detection path. 


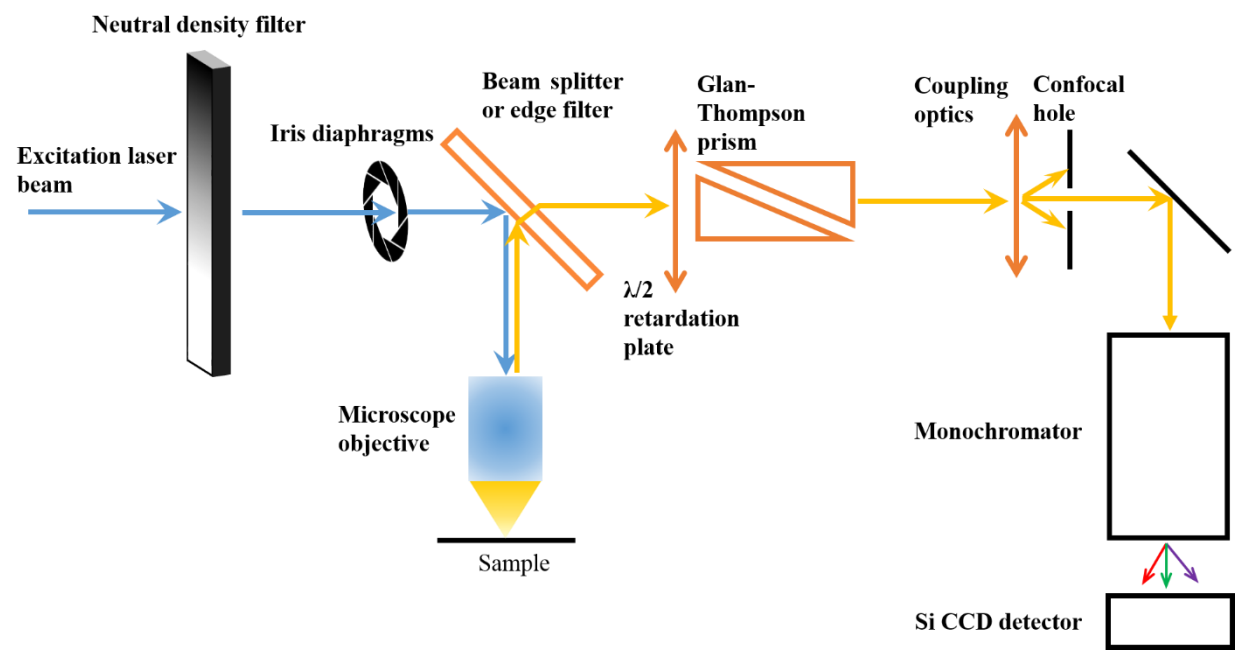

Figure 11. Schematic diagram of the experimental setup employed in this work for $\mu$-PL/Raman/PLE measurements.

\subsection{Micro-photoluminescence excitation spectroscopy.}

In PL experiments monitored radiative transitions usually originate from the lowest excited states of an electronic system under consideration. Contributions of emissions that stem from higher laying excited states is typically negligible due to fast energy relaxation of the photo generated carriers to the lower energy levels. However information regarding the excited states can be gained by monitoring the PL intensity as a function of photon excitation energy, i.e. using the photoluminescence excitation (PLE) spectroscopy. This is because the monitored PL intensity increases when the laser energy is tuned in resonance with an excited state of the electronic system, due to resonant absorption the photon.

The experimental setup for the $\mu$-PLE measurements is essentially the same as that used for the $\mu$-PL measurements with the only difference that a tunable Ti:sapphire laser is used. Since the PL signal is detected by a Si CCD matrix detector, PLE spectra can be simultaneously acquired for a range of the emission energies. In practice this is done by measuring temporal evolution of the PL spectra with equally distant time intervals while the excitation energy is slowly scanned in time. Since the laser power changes at different excitation energies, the laser power profile is recorded simultaneously in order to correct consequent variations in the monitored PL intensity. 


\subsection{Micro-Raman spectroscopy.}

Raman spectroscopy is a powerful and non-destructive tool for structural and electronic characterization of materials such as presence of crystal disorder, presence of defects, etc. Basic physical principles of the Raman scattering were already discussed in the chapter 2.

In an inelastic light scattering technique the spectrum of the scattered light is recorded. It consists of the scattered light beam and the useful signal. Whereas the former is very strong and can even destroy a detector, the latter is relatively weak. Since typical optical phonon energies accessible via inelastic light scattering are tens of $\mathrm{meV}$, a rejection filter with extremely sharp cut-off is needed in order to eliminate the reflected laser light. The rejection of the reflected beam can be performed with long-pass edge filters. These filters have optical density $(\mathrm{OD})>6$ in the rejecting region allowing to detect the Raman signal. However these filters have strict requirements on the incidence angle. Typically they allow variation of the angle by few degrees. This is accompanied by a shift of the cut off frequency away from the "specified" one by a small amount.

Micro-Raman measurements were performed on the same setup as used for the $\mu-P L / \mu-$ PLE experiments and is shown schematically in Figure 11. The modification from PL/PLE mode is done by exchanging the beam splitter by the edge filter of the corresponding cut-off wavelength. The edge filters are fixed on a kinematic mount which allows to adjust an angle of incidence of the laser beam. 


\section{Bibliography.}

[1] Figure taken from $h t t p: / / w w w . s e a s . u p e n n . e d u / \sim e s e 218 / s 04 / h w s-S 04 / h w 1 / h w 1-s o l . h t m l$.

[2] Figure taken from https://en.wikipedia.org/wiki/Hexagonal_crystal_system.

[3] D. J. Chadi and M. L. Cohen, Phys. Status Solidi 68, 405 (1975).

[4] W. A. Harrison, Electronic Structure and the Properties of Solids: The Physics of the Chemical Bond (Dover publications Inc., New York, 1989).

[5] Y. Sun, S. E. Thompson, and T. Nishida, Strain Effect in Semiconductors: Theory and Device Applications (Springer, 2010), p. 353.

[6] M. S. Dresselhaus, G. Dresselhaus, and A. Jorio, Group Theory: Application to the Physics of Condensed Matter, 1st ed. (Springer Berlin Heidelberg, Berlin, Heidelberg, 2008), pp. XV, 582.

[7] A. De and C. E. Pryor, Phys. Rev. B 81, 155210 (2010).

[8] J. L. Birman, Phys. Rev. Lett. 2, 157 (1959).

[9] J. L. Birman, Phys. Rev. Lett. 114, 1490 (1959).

[10] G. F. Koster, The Properties of the Thirty-Two Point Groups (M.I.T. Press, Cambridge, 1963), p. 104.

[11] L. C. L. Y. Voon and M. Willatzen, The KP Method: Electronic Properties of Semiconductors (Springer, 2009), p. 446.

[12] A. A. Maradudin, E. W. Montroll, and G. H. Weiss, Theory of Lattice Dynamics in the Harmonic Approximation (Academic Press, New York, 1963), p. 319.

[13] H. Poulet and J. P. Mathieu, Vibration Spectra and Symmetry of Crystals (Gordon and Breach, Science Publishers, 1976), p. 656.

[14] Zardo, S. Conesa-Boj, F. Peiro, J.R. Morante, J. Arbiol, E. Uccelli, G. Abstreiter, and A. Fontcuberta i Morral, Phys. Rev. B 80, 245324 (2009).

[15] S. Crankshaw, L. C. Chuang, M. Moewe, and C. Chang-Hasnain, Phys. Rev. B 81, 233303 (2010).

[16] C. H. Park, B. Cheong, K. Lee, and K. J. Chang, Phys. Rev. B 49, (1994).

[17] C.-Y. Yeh, Z. W. Lu, S. Froyen, and A. Zunger, Phys. Rev. B 45, 12130 (1992).

[18] C. A. Arguello, D. L. Rousseau, and S. P. S. Porto, Phys. Rev. 423, 1351 (1969).

[19] R. Loudon, Adv. Phys. 13, 423 (1964).

[20] R. Durman, P. Favre, U.A. Jayasooriya, and S. F. A. Kettle, J. Crystallogr. Spectrosc. Res. 17, 431 (1987).

[21] G. Burns, F. H. Dacol, C. R. Wie, E. Bursteint, and M. Cardona, Solid 62, 449 (1987).

[22] J. Ibáñez, E. Alarcón-Lladó, R. Cuscó, L. Artús, and M. Hopkinson, J. Appl. Phys. 102, 
013502 (2007).

[23] M. Born and K. Huang, Dynamical Theory of Crystal Lattices (Oxford University Press, Oxford, 1954), p. 421.

[24] F. Cerdeira, C. J. Buchenauer, F. H. Pollak, and M. Cardona, Phys. Rev. B 5, 580 (1972).

[25] W. Pötz and P. Vogl, Phys. Rev. B 24, 2025 (1981).

[26] A. Blacha, H. Presting, and M. Cardona, Phys. Status Solidi 126, 11 (1984).

[27] C. Trallero-Giner, A. Cantarero, M. Cardona, and M. Mora, Phys. Rev. B 45, 6061 (1992).

[28] E. Anastassakis, A. Pinczuk, E. Burstein, F. H. Pollak, M. Cardona, and R. Island, Solid State Commun. 8, 133 (1970).

[29] T. Prokofyeva, T. Sauncy, M. Seon, M. Holtz, Y. Qiu, S. Nikishin, and H. Temkin, Appl. Phys. Lett. 73, 1409 (1998).

[30] G. Lucovsky, M. H. Brodsky, and E. Burstein, Phys. Rev. B 2, 3295 (1970).

[31] I. F. Chang and S. S. Mitra, Phys. Rev. 172, 924 (1968).

[32] L. Genzel, T. P. Martin, and C. H. Perry, Phys. Status Solidi 62, 83 (1974).

[33] R. Fuchs and K. L. Kliewer, Phys. Rev. 140, A2076 (1965).

[34] Q. H. Xiong, R. Gupta, J. Wang, G. D. Mahan, L. C. L. Y. Voon, and P. C. Eklund, Solid State Phenom. 121-123, 955 (2007).

[35] V. A. Fonoberov and A. A. Balandin, Phys. Rev. B 70, 233205 (2004).

[36] P.-M. Chassaing, F. Demangeot, V. Paillard, A. Zwick, N. Combe, C. Pagès, M. L. Kahn, A. Maisonnat, and B. Chaudret, Phys. Rev. B 77, 153306 (2008).

[37] P.-M. Chassaing, F. Demangeot, V. Paillard, A. Zwick, N. Combe, C. Pagès, M.L. Kahn, A. Maisonnat, and B. Chaudret, Appl. Phys. Lett. 91, 053108 (2007).

[38] H. F. Liu, S. Tripathy, G. X. Hu, and H. Gong, J. Appl. Phys. 105, 053507 (2009).

[39] R. S. Knox, Theory of Excitons (Press, Academic, New York, 1963), p. 207.

[40] K. Cho, Phys. Rev. B 14, 4463 (1976).

[41] K. Cho, ed. Excitons (Springer-Verlag Berlin Heidelberg, 1979), p. 275.

[42] D. G. Thomas and J. J. Hopfield, Phys. Rev. 150, 680 (1966).

[43] H. Mathinieu, L. Bayo, J. Camassel, and P. Merle, Phys. Rev. B 22, 4834 (1980).

[44] B. Gil, J. Camassel, P. Merle, and H. Mathieu, Phys. Rev. B 25, 3987 (1982).

[45] B. Gil, J. Camassel, J.P. Albert, and H. Mathieu, Phys. Rev. B 33, 2690 (1986).

[46] B. Gil and H. Mariette, Phys. Rev. B 35, 7999 (1987).

[47] S. Francoeur and S. Marcet, J. Appl. Phys. 108, 043710 (2010).

[48] L. Bergman and J. L. McHale, ed. Handbook of Luminescent Semiconductor Materials 
(CRC Press, 2012), p. 437.

[49] E. Poem, J. Shemesh, I. Marderfeld, D. Galushko, N. Akopian, D. Gershoni, B. D. Gerardot, A. Badolato, and P. M. Petroff, Phys. Rev. B 76, 235304 (2007).

[50] A. Franceschetti, L. W. Wang, H. Fu, and A. Zunger, Phys. Rev. B 58, R13367 (1998).

[51] A. J. Williamson, L. W. Wang, and A. Zunger, Phys. Rev. B 62, 963 (2000).

[52] J.-W. Luo, G. Bester, and A. Zunger, New J. Phys. 11, 123024 (2009).

[53] S. Lee, L. Jönsson, J. W. Wilkins, G. W. Bryant, and G. Klimeck, Phys. Rev. B 63, 195318 (2001).

[54] M. Zieliński, M. Korkusiński, and P. Hawrylak, Phys. Rev. B 81, 085301 (2010).

[55] T. Takagahara, Phys. Rev. B 47, 4569 (1993).

[56] T. Takagahara, Phys. Rev. B 62, 16840 (2000).

[57] H. Tong and M. W. Wu, Phys. Rev. B 83, 235323 (2011).

[58] E. Kadantsev and P. Hawrylak, Phys. Rev. B 81, 045311 (2010).

[59] E. Tsitsishvili, R. V. Baltz, and H. Kalt, Phys. Rev. B 67, 205330 (2003).

[60] E. Tsitsishvili, R. V. Baltz, and H. Kalt, Phys. Status Solidi 1555, 1552 (2003).

[61] G. Bester, S. Nair, and A. Zunger, Phys. Rev. B 67, 161306 (2003).

[62] R. Singh and G. Bester, Phys. Rev. Lett. 103, 063601 (2009).

[63] G. Bester and A. Zunger, Phys. Rev. B 71, 045318 (2005).

[64] A. Schliwa, M. Winkelnkemper, and D. Bimberg, Phys. Rev. B 76, 205324 (2007).

[65] G. Bester, A. Zunger, X. Wu, and D. Vanderbilt, Phys. Rev. B 74, 081305 (2006).

[66] O. Stier, M. Grundmann, and D. Bimberg, Phys. Rev. B 59, 5688 (1999).

[67] R. Seguin, A. Schliwa, S. Rodt, K. Pötschke, U.W. Pohl, and D. Bimberg, Phys. Rev. Lett. 95, 257402 (2005).

[68] V. P. LaBella, H. Yang, D.W. Bullock, P. M. Thibado, P. Kratzer, and M. Scheffler, Phys. Rev. Lett. 83, 2989 (1999).

[69] M. Abbarchi, C. A. Mastrandrea, T. Kuroda, T. Mano, K. Sakoda, N. Koguchi, S. Sanguinetti, A. Vinattieri, and M. Gurioli, Phys. Rev. B 78, 125321 (2008).

[70] L. Besombes, K. Kheng, and D. Martrou, Phys. Rev. Lett. 85, 425 (2000).

[71] R. J. Young, R. M. Stevenson, A. J. Shields, P. Atkinson, K. Cooper, D. A. Ritchie, K. M. Groom, A. I. Tartakovskii, and M.S. Skolnick, Phys. Rev. B 72, 113305 (2005).

[72] J. D. Plumhof, V. Křápek, L. Wang, A. Schliwa, D. Bimberg, A. Rastelli, and O. G. Schmidt, Phys. Rev. B 81, 121309 (2010).

[73] V. Mlinar and A. Zunger, Phys. Rev. B 79, 115416 (2009).

[74] L. O. Mereni, O. Marquardt, G. Juska, V. Dimastrodonato, E. P. O’Reilly, and E. 
Pelucchi, Phys. Rev. B 85, 155453 (2012).

[75] J. Wang, M. S. Gudiksen, X. Duan, Y. Cui, and C. M. Lieber, Science 293, 1455 (2001).

[76] A. Lan, J. Giblin, V. Protasenko, and M. Kuno, Appl. Phys. Lett. 92, 183110 (2008).

[77] G. Zhang, K. Tateno, H. Sanada, T. Tawara, H. Gotoh, and H. Nakano, Appl. Phys. Lett. 95, 123104 (2009).

[78] H. E. Ruda and A. Shik, J. Appl. Phys. 100, 024314 (2006).

[79] C. Wilhelm, A. Larrue, X. Dai, D. Migas, and C. Soci, Nanoscale 4, 1446 (2012).

[80] A. Mishra, L. V. Titova, T. B. Hoang, H. E. Jackson, L. M. Smith, J. M. Yarrison-Rice, Y. Kim, H. J. Joyce, Q. Gao, H. H. Tan, and C. Jagadish, Appl. Phys. Lett. 91, 263104 (2007).

[81] Y. Masumoto, Y. Hirata, P. Mohan, J. Motohisa, and T. Fukui, Appl. Phys. Lett. 98, 211902 (2011).

[82] L. Ahtapodov, J. Todorovic, P. Olk, T. Mjåland, P. Slåttnes, D. L. Dheeraj, A. T. J. van Helvoort, B.-O. Fimland, and H. Weman, Nano Lett. 12, 6090 (2012).

[83] T. Ba Hoang, A. F. Moses, L. Ahtapodov, H. Zhou, D. L. Dheeraj, A. T. J. van Helvoort, B.-O. Fimland, and H. Weman, Nano Lett. 10, 2927 (2010).

[84] K. Pemasiri, M. Montazeri, R. Gass, L.M. Smith, H. E. Jackson, J. Yarrison-Rice, S. Paiman, Q. Gao, H. H. Tan, C. Jagadish, X. Zhang, and J. Zou, Nano Lett. 9, 648 (2009).

[85] J. Bao, D. C. Bell, F. Capasso, J. B. Wagner, T. Mårtensson, J. Trägårdh, and L. Samuelson, Nano Lett. 8, 836 (2008).

[86] E. G. Gadret, G. O. Dias, L. C. O. Dacal, M. M. de Lima, C. V. R. S. Ruffo, F. Iikawa, M. J. S. P. Brasil, T. Chiaramonte, M. A. Cotta, L. H. G. Tizei, D. Ugarte, and A. Cantarero, Phys. Rev. B 82, 125327 (2010).

[87] P. Kusch, E. Grelich, C. Somaschini, E. Luna, M. Ramsteiner, L. Geelhaar, H. Riechert, and S. Reich, Phys. Rev. B 89, 045310 (2014).

[88] A. Mukherjee, S. Ghosh, S. Breuer, U. Jahn, L. Geelhaar, and H. T. Grahn, J. Appl. Phys. 117, 054308 (2015).

[89] N. Vainorius, D. Jacobsson, S. Lehmann, A. Gustafsson, K. A. Dick, L. Samuelson, and M.-E. Pistol, Phys. Rev. B 89, 165423 (2014).

[90] U. Jahn, J. Lähnemann, C. Pfüller, O. Brandt, S. Breuer, B. Jenichen, M. Ramsteiner, L. Geelhaar, and H. Riechert, Phys. Rev. B 85, 045323 (2012).

[91] U. Ozzgür, Y. I. Alivov, C. Liu, A. Teke, M. A. Reshchikov, S. Dogă, V. Avrutin, S.-J. Cho, and H. Morkoc, J. Appl. Phys. 98, 041301 (2005).

[92] W. R. L. Lambrecht, A. V. Rodina, S. Limpijumnong, B. Segall, and B. K. Meyer, Phys. Rev. B 65, 075207 (2002). 
[93] V. Rodina, M. Strassburg, M. Dworzak, U. Haboeck, A. Hoffmann, A. Zeuner, H. R. Alves, D. M. Hofmann, and B. K. Meyer, Phys. Rev. B 69, 125206 (2004).

[94] M. R. Wagner, J.-H. Schulze, R. Kirste, M. Cobet, A. Hoffmann, C. Rauch, A. V. Rodina, B. K. Meyer, U. Röder, and K. Thonke, Phys. Rev. B 80, 205203 (2009).

[95] S. Adachi, Physical Properties of III-V Semiconductor Compounds: InP, InAs, GaAs, GaP, In GaAs, and InGaAsP (John Wiley\&Sons, 1992), p. 318.

[96] S. Adachi, Properties of Group-IV, III-V and II-VI Semiconductors (John Wiley\&Sons, 2005), p. 387.

[97] I. A. Buyanova and W.M. Chen, editors, Physics and Applications of Dilute Nitrides (Taylor\&Francis, 2004), p. 477.

[98] W. Shan, W. Walukiewicz, J. W. Ager, E. E. Haller, J.F. Geisz, D. J. Friedman, J. M. O1son, and S. R. Kurtz, Phys. Rev. Lett. 82, 1221 (1999).

[99] W. Shan, W. Walukiewicz, K. M. Yu, J. Wu, J. W. Ager, E. E. Haller, H. P. Xin, and C. W. Tu, Appl. Phys. Lett. 76, 3251 (2000).

[100] I. A. Buyanova, M. Izadifard, W. M. Chen, H. P. Xin, and C. W. Tu, Phys. Rev. B 69, 201303 (2004).

[101] G. Leibiger, V. Gottschalch, M. Schubert, G. Benndorf, and R. Schwabe, Phys. Rev. B 65, 245207 (2002).

[102] I. A. Buyanova, M. Izadifard, A. Kasic, H. Arwin, W.M. Chen, H. P. Xin, Y. G. Hong, and C. W. Tu, Phys. Rev. B 70, 085209 (2004).

[103] P. R. C. Kent and A. Zunger, Phys. Rev. B 64, 115208 (2001).

[104] A. Lindsay and E. P. O'Reilly, Phys. Rev. Lett. 93, 196402 (2004).

[105] E. P. O’Reilly, A. Lindsay, S. Tomi, and M. Kamal-Saadi, Semicond. Sci. Technol. 17, 870 (2002).

[106] I. A. Buyanova, G. Y. Rudko, W. M. Chen, H. P. Xin, and C. W. Tu, Appl. Phys. Lett. 80, 1740 (2002).

[107] C. Harris, A. Lindsay, and E.P. O’Reilly, J. Phys. Condens. Matter 20, 295211 (2008). 


\section{Papers}

The articles associated with this thesis have been removed for copyright reasons. For more details about these see:

http://urn.kb.se/resolve?urn=urn:nbn:se:liu:diva-123939 